\title{
Science with the TianQin Observatory: Preliminary results on Galactic double white dwarf binaries
}

\author{
Shun-Jia Huang, ${ }^{1}$ Yi-Ming Hu, ${ }^{1, *}$ Valeriya Korol, ${ }^{2}$ Peng-Cheng Li, ${ }^{3,4,1}$ Zheng-Cheng \\ Liang, ${ }^{5,1}$ Yang Lu, ${ }^{1}$ Hai-Tian Wang,,${ }^{6,1}$ Shenghua Yu, ${ }^{8,9}$ and Jianwei Mei ${ }^{1, \dagger}$ \\ ${ }^{1}$ TianQin Research Center for Gravitational Physics and School of Physics and Astronomy, \\ Sun Yat-sen University (Zhuhai Campus), Zhuhai 519082, People's Republic of China \\ ${ }^{2}$ School of Physics and Astronomy, University of Birmingham, Birmingham B15 2TT, United Kingdom \\ ${ }^{3}$ Center for High Energy Physics, Peking University, \\ No. 5 Yiheyuan Road, Beijing 100871, People's Republic of China \\ ${ }^{4}$ Department of Physics and State Key Laboratory of Nuclear Physics and Technology, \\ Peking University, No. 5 Yiheyuan Road, Beijing 100871, People's Republic of China \\ ${ }^{5}$ MOE Key Laboratory of Fundamental Physical Quantities Measurements, Hubei Key \\ Laboratory of Gravitation and Quantum Physics, PGMF and School of Physics, Huazhong \\ University of Science and Technology, Wuhan 430074, People's Republic of China \\ ${ }^{6}$ Purple Mountain Observatory, Chinese Academy of Sciences, Nanjing 210023, People's Republic of China \\ ${ }^{7}$ School of Astronomy and Space Science, University of Science and Technology of China, \\ Hefei, Anhui 230026, People's Republic of China \\ ${ }^{8}$ National Astronomical Observatories, Chinese Academy of Sciences, Beijing 100012, People's Republic of China \\ ${ }^{9}$ The Key Laboratory of Radio Astronomy, Chinese Academy of Sciences, Beijing 100012, People's Republic of China
}

(Dated: September 25, 2020)

\begin{abstract}
We explore the prospects of detecting Galactic double white dwarf (DWD) binaries with the space-based gravitational wave (GW) observatory TianQin. In this work, we analyze both a sample of currently known DWDs and a realistic synthetic population of DWDs to assess the number of guaranteed detections and the full capacity of the mission. We find that TianQin can detect 12 out of $\sim 100$ known DWDs; GW signals of these binaries can be modeled in detail ahead of the mission launch, and therefore they can be used as verification sources. Besides, we estimate that TianQin has a potential to detect as many as $10^{4}$ DWDs in the Milky Way. TianQin is expected to measure their orbital periods and amplitudes with accuracies of $\sim 10^{-7}$ and $\sim 0.2$, respectively, and to localize on the sky a large fraction (39\%) of the detected population to better than $1 \operatorname{deg}^{2}$. We conclude that TianQin has the potential to significantly advance our knowledge on Galactic DWDs by increasing the sample up to 2 orders of magnitude, and will allow their multi-messenger studies in combination with electromagnetic telescopes. We also test the possibilities of different configurations of TianQin: (1) the same mission with a different orientation, (2) two perpendicular constellations combined into a network, and (3) the combination of the network with the ESA-led Laser Interferometer Space Antenna. We find that the network of detectors boosts the accuracy on the measurement of source parameters by 1-2 orders of magnitude, with the improvement on sky localization being the most significant.
\end{abstract}

\section{INTRODUCTION}

The first direct detection of gravitational waves (GWs) generated from a binary black hole merger (GW150914) was made by the LIGO and Virgo Collaborations in 2015 [1], one hundred years after they were predicted by Albert Einstein [2]. This detection, together with several subsequent ones including a binary neutron star merger (GW170817), started new fields of GW and multimessenger astronomy [3-7].

The sensitivity band of the currently operational ground-based detectors LIGO and Virgo is limited between $10 \mathrm{~Hz}$ and kilohertz frequencies [8]. However, GW sources span many orders of magnitude in frequency down to femtohertz. Several experiments aim to cover such a large spectrum: the cosmic microwave background

\footnotetext{
* huyiming@mail.sysu.edu.cn

$\dagger$ meijw@sysu.edu.cn
}

polarization experiments [9], the pulsar timing array $[10,11]$, and the space-based laser interferometers, sensitive to femtohertz, nanohertz and millihertz frequencies, respectively $[12,13]$.

The millihertz frequency band is populated by a large variety of GW sources: massive black hole binaries $\left(10^{3}\right.$ $10^{7} \mathrm{M}_{\odot}$ ) formed via galaxy mergers [14-18]; compact stellar objects orbiting massive black holes, called extreme mass ratio inspirals (EMRIs) [19, 20]; ultra-compact stellar mass binaries (and multiples) composed of white dwarfs, neutron stars and stellar-mass black holes in the neighborhood of the Milky Way [21-24]. Besides individually resolved binaries, stochastic backgrounds of astrophysical and cosmological origin can be detected at millihertz frequencies [e.g. 25, 26]. Therefore, this band is expected to provide rich and diverse science, ranging from Galactic astronomy to high-redshift cosmology and to fundamental physics [27-31].

Among all kinds of ultra-compact stellar mass binaries, those composed of two white dwarf stars [double 
white dwarf binaries (DWDs)] comprise the absolute majority (up to $10^{8}$ ) in the Milky Way. Being abundant and nearby, DWDs are expected to be the most numerous GW sources for space-based detectors [21, 32-34].

Individual GW detections of DWDs will significantly advance our knowledge on binary formation and white dwarf stars themselves in a number of ways. First, DWDs represent the end products of the low-mass binary evolution, and as such they encode information on physical processes such as the highly uncertain mass transfer and common envelope phases [35, 36]. Second, DWDs are progenitors to AM canum venaticorum (AM CVn) systems, short-period ( $\lesssim 1$ hour) mass-transferring DWDs, ideal for studying the stability of the mass transfer [3740]. Third, DWD mergers are thought to originate a broad range of interesting transient events including type-Ia supernovae (SNe Ia) [41-43]. In addition, detached DWDs are particularly suitable for studying the physics of tides. DWDs affected by tides will yield information on the nature and origin of white dwarf viscosity, which is still a missing piece in our understanding of white dwarfs' interior matter [44-47]. Finally, by analyzing their GW signals one could set constraints on deviations from general relativity $[48,49]$.

The overall GW signal from DWDs imprints the information on the Galactic stellar population as a whole, and it can constrain the structural properties of the Milky Way [34, 50-53]. A significant fraction of the population may present a stellar or sub-stellar tertiary companions, that can be recognized by an extra frequency modulation of the DWD GW signals [24, 31, 54]. GW detectors have the potential to guide the discovery of these populations [55].

TianQin is a space-based GW observatory sensitive to millihertz frequencies [13, 56, 57]. Recently, a significant effort has been put into the study and consolidation of the science cases for TianQin [58]. On the astrophysics side, these efforts include studies on the detection prospect of massive black hole binaries [15, 59], EMRIs [20], stellarmass black hole binaries [60], and stochastic backgrounds [26]; on the fundamental physics side, prospects for testing of the no-hair theorem with GWs from massive black hole binaries [29] and constraints on modified gravity theories [30, 61-63] have been assessed for TianQin. In this paper, we aim to forecast the detection of Galactic DWDs with TianQin. Due to their low masses, the GW horizon of DWDs is limited within the Milky Way, possibly reaching nearby satellite galaxies and the Andromeda galaxy $[21,27,64,65]$. Therefore, in this study we focus on the Galactic population only. We concentrate on detached systems, because they are expected to be orders of magnitude more numerous than other types of binaries in the millihertz frequency regime [e.g., 66, 67].

The paper is organized as follows. In Section II, we outline the sample of the currently known ultra-compact DWDs and AM CVn's, and we present a mock Galactic population. In Section III, we derive analytical expressions for computing the signal-to-noise ratio and uncer- tainties on binary parameters for TianQin. In Section IV, we present our results on the detectability of the known DWDs and that of the mock population. We also present similar results for some mission variations and explore the improvements that could be achieved when a few detectors work as a network. Finally, we summarize our main findings in Section V.

\section{GALACTIC DOUBLE WHITE DWARF BINARIES}

The currently known electromagnetic (EM) sample amounts to $\sim 100$ detached and $\sim 60$ interacting (AM CVn) DWD systems with orbital periods $\lesssim 1$ day [68-70]. Although rapidly expanding with several recent detections [71-74], this sample is still limited and represents only the tip of the iceberg of the overall Galactic population. To quantify the ability of TianQin in detecting DWDs, in this study we consider both the known sample and a synthetic Galactic population. In this section, we briefly outline both samples.

\section{A. Candidate verification binaries}

Binaries discovered through EM observations are often called verification binaries in the literature [e.g. 75, 76]. This is because we can measure their parameters and therefore accurately model their GW signals; the predicted signal can be used to verify the detector's performance. Here we consider a sample of 81 candidate verification binaries (CVBs) (40 AM CVn type systems and 41 detached DWDs) with orbital periods $\lesssim 5$ hours. FIG. 1 shows the sky positions and the luminosity distances of our CVBs in the ecliptic coordinate system.

We list parameters of verification binaries in Table $\mathrm{V}$ in Appendix A. Parameters with poor observational constraints have been inferred from theoretical models. For example, for most verification binaries, trigonometric parallaxes from Gaia Data Release 2 [77] can be used to determine their luminosity distance [76]. Distances to RX J0806.3+1527 (also known as HM Cancri, hereafter J0806 [78]), CR Boo, V803 Cen, SDSS J093506.92+441107.0, SDSS J075552.40+490627.9, SDSS J002207.65-101423.5 and SDSS J110815.50+151246.6, however, are determined using different methods. In particular, J0806 has a largely uncertain distance. Here we use a conservative upper boundary of $5 \mathrm{kpc}$ based on its luminosity observation [79].

In this work we define a DWD system as a verification binary if (1) it has been detected in the electromagnetic (EM) bands, and (2) its expected GW signal-to-noise ratio (SNR) for TianQin is $\geq 5$ with a nominal mission lifetime of five years $[75,76]$. We adopt a relatively low SNR threshold for the detection of the verification binaries, because there is a priori information from the EM 


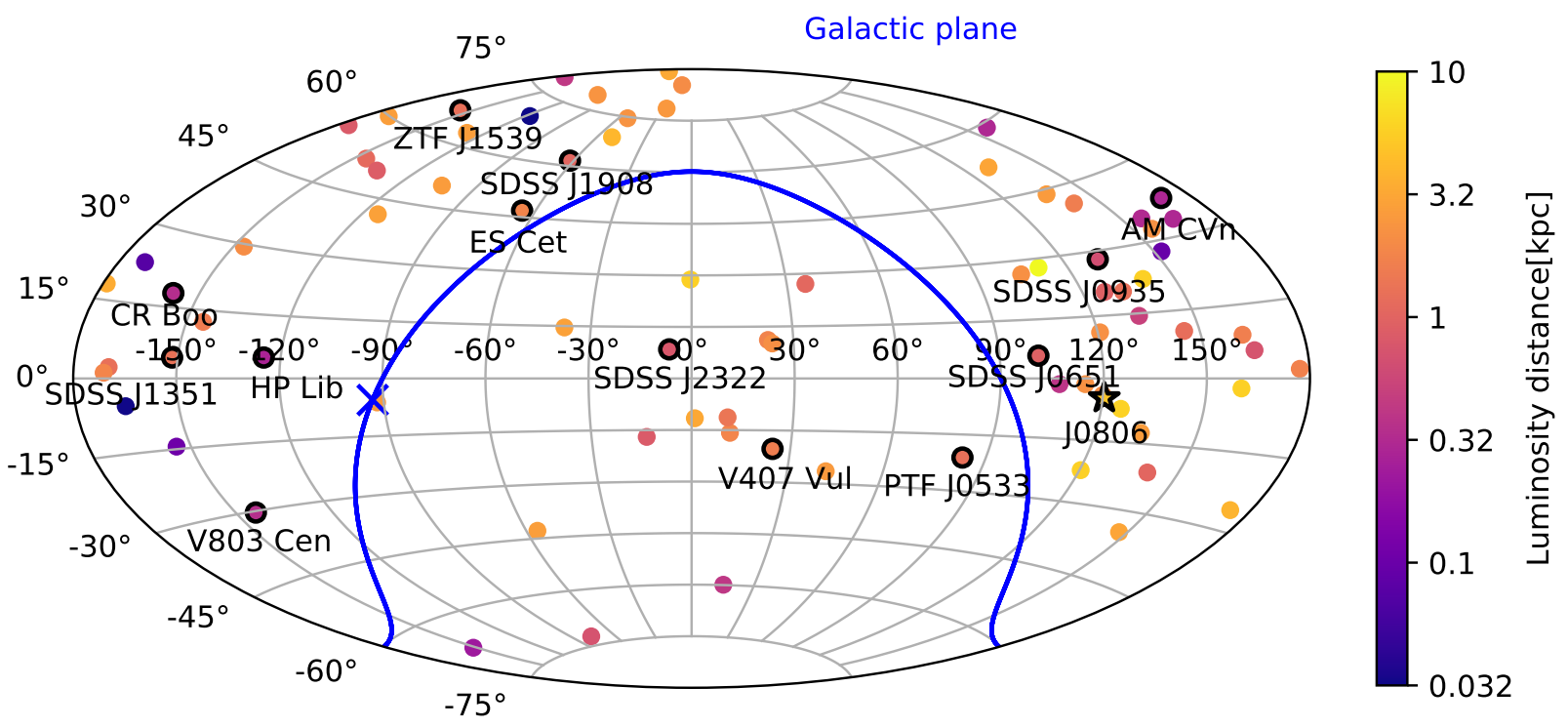

FIG. 1. Sky positions of the 81 candidate verification binaries shown in the ecliptic coordinate system, with lighter colors representing shorter distances to the Solar System. Binaries with the highest SNR are highlighted. The blue line indicates the Galactic plane, with the Galactic Center marked by the blue cross.

observations to fall back on. We also define the potential verification binaries to be the CVBs which have $3 \leq \mathrm{SNR}$ $<5[75,76]$.

\section{B. Synthetic Galactic population}

In this study, we employ a synthetic catalog of Galactic DWDs based on models of Toonen et al. [80, 81]. These models are constructed on a statistically significant number of progenitor zero-age main sequence systems $\left(\sim 10^{5}\right)$ evolved with binary population synthesis code SEBA [82] until both stars become white dwarfs. To construct the progenitor population the mass of the primary star is drawn from the Kroupa initial mass function in the range between 0.95 and $10 \mathrm{M}_{\odot}$ [83]. Then, the mass of the secondary is drawn from a uniform mass ratio distribution between 0 and 1 [84]. Orbital separations and eccentricities are obtained from a the log-flat distribution (considering those binaries that on the zero-age main sequence have orbital separations up to $10^{6} R_{\odot}$.) and a thermal distribution, respectively [84-86]. The binary fraction is set to $50 \%$ and the metallicity to solar. It is important to note that in this paper we use models that employ the $\alpha \gamma$-common envelope evolution model designed and fine-tuned on observed DWDs [37, 87]. We highlight that this model matches well the mass ratio distribution [80] and the number density [81] of the observed DWDs.

Next, we assign the spatial and the age distributions to synthetic binaries. Specifically, we use a smooth Milky Way potential consistent of an exponential stellar disc and a spherical central bulge, adopting scale parameters as in [52, see table 1 of that source]. The stellar density distribution is normalized according to the star formation history numerically computed by Boissier and Prantzos [88], while the age of the Galaxy is set to $13.5 \mathrm{Gyr}$. We account for the change in binary orbital periods due to GW radiation from the moment of DWD formation until 13.5 Gyr.

Finally, for each binary we assign an inclination angle $\iota$, drawn randomly from a uniform distribution in $\cos \iota$. The polarization angle and the initial orbital phase $\left(\psi_{S}\right.$ and $\phi_{0}$, respectively) are randomized, assuming uniform distribution over the intervals of $[0, \pi)$ and $[0,2 \pi)$, respectively. The obtained catalog contains the following parameters: orbital period $P$, component masses $m_{1}$ and $m_{2}$, the ecliptic latitude $\lambda$ and longitude $\beta$, distance from the Sun $d$, and angles $\iota, \psi_{S}, \phi_{0}$. This catalog has been originally employed in the study of DWD detectability with LISA [12]. Therefore, this paper represents a fair comparison with the results in Amaro-Seoane et al. [12]. 


\section{SIGNAL AND NOISE MODELING}

\section{A. Gravitational wave signals from a monochromatic source}

The timescale on which DWDs' orbits shrink via GW radiation is typically $>\mathrm{Myr}$ (at low frequencies). This is significantly greater than the mission lifetime of TianQin of several years; the two timescales are only comparable when $f / \dot{f} \sim T_{\mathrm{m}}$ :

$$
f=0.18\left(\frac{T_{\mathrm{m}}}{5 \mathrm{yr}}\right)^{-3 / 8}\left(\frac{\mathcal{M}}{1 M_{\odot}}\right)^{-5 / 8} \mathrm{~Hz}
$$

Therefore, binaries with frequencies significantly smaller than $0.18 \mathrm{~Hz}$ can be safely considered as monochromatic GW sources, meaning that they can be described by a set of seven parameters: the dimensionless amplitude $(\mathcal{A})$, GW frequency $f=2 / P, \lambda, \beta, \iota, \psi_{S}$ and $\phi_{0}$. Note that we do not include eccentricity because DWDs circularize during the common envelope phase.

GWs emitted by a monochromatic source can be computed using the quadrupole approximation [89, 90]. In this approximation, the GW signal can be described as a combination of the two polarizations $(+$ and $\times)$ :

$$
\begin{gathered}
h_{+}(t)=\mathcal{A}\left(1+\cos \iota^{2}\right) \cos \left(2 \pi f t+\phi_{0}+\Phi_{D}(t)\right), \\
h_{\times}(t)=2 \mathcal{A} \cos \iota \sin \left(2 \pi f t+\phi_{0}+\Phi_{D}(t)\right),
\end{gathered}
$$

with

$$
\mathcal{A}=\frac{2(G \mathcal{M})^{5 / 3}}{c^{4} d}(\pi f)^{2 / 3}
$$

where $\mathcal{M} \equiv\left(m_{1} m_{2}\right)^{3 / 5} /\left(m_{1}+m_{2}\right)^{1 / 5}$ is the chirp mass, and $G$ and $c$ are the gravitational constant and the speed of light, respectively. Note that the additional term $\Phi_{D}(t)$ in the GW phase [Eq. (2)-(3)] is the Doppler phase arising from the periodic motion of TianQin around the Sun:

$$
\Phi_{D}(t)=2 \pi f t \frac{R}{c} \sin (\pi / 2-\beta) \cos \left(2 \pi f_{m} t-\lambda\right),
$$

where $R=1 \mathrm{~A} . \mathrm{U}$. is the distance between the Earth and the Sun, and $f_{m}=1$ /year is the modulation frequency. $\lambda$ and $\beta$ are the ecliptic coordinates of the source.

\section{B. Detector's response to GW signals}

The design of the TianQin mission [13] envisions a constellation of three drag-free satellites orbiting the Earth, maintaining a distance between each other of $\sim 10^{5} \mathrm{~km}$. Satellites will form an equilateral triangle constellation oriented in such a way that the normal vector to the detector's plane is pointing towards J0806 $\left(\lambda=120.4^{\circ}\right.$, $\left.\beta=-4.7^{\circ}\right)$.

In the low-frequency limit $\left(f \ll f_{*}\right.$ with $f_{*}=c / 2 \pi L$ being the transfer frequency, $\sim 0.28 \mathrm{~Hz}$ for TianQin), the GW strain recorded by the detector can be described as a linear combination of the two GW polarizations modulated by the detector's response [91]:

$$
h(t)=h_{+}(t) F^{+}(t)+h_{\times}(t) F^{\times}(t),
$$

where $F^{+, \times}(t)$ are the antenna pattern functions.

For a detector with an equilateral triangle geometry, two orthogonal Michelson signals can be constructed and the antenna pattern functions can be expressed as

$$
\begin{aligned}
& F_{1}^{+}\left(t, \theta_{S}, \phi_{S}, \psi_{S}\right)=\frac{\sqrt{3}}{2}\left(\frac{1}{2}\left(1+\cos ^{2} \theta_{S}\right) \cos 2 \phi_{S}(t) \cos 2 \psi_{S}-\cos \theta_{S} \sin 2 \phi_{S}(t) \sin 2 \psi_{S}\right), \\
& F_{1}^{\times}\left(t, \theta_{S}, \phi_{S}, \psi_{S}\right)=\frac{\sqrt{3}}{2}\left(\frac{1}{2}\left(1+\cos ^{2} \theta_{S}\right) \cos 2 \phi_{S}(t) \sin 2 \psi_{S}+\cos \theta_{S} \sin 2 \phi_{S}(t) \cos 2 \psi_{S}\right), \\
& F_{2}^{+}\left(t, \theta_{S}, \phi_{S}, \psi_{S}\right)=F_{1}^{+}\left(t, \theta_{S}, \phi_{S}-\frac{\pi}{4}, \psi_{S}\right), \\
& F_{2}^{\times}\left(t, \theta_{S}, \phi_{S}, \psi_{S}\right)=F_{1}^{\times}\left(t, \theta_{S}, \phi_{S}-\frac{\pi}{4}, \psi_{S}\right),
\end{aligned}
$$

where $\sqrt{3} / 2$ represents a factor originating from the geometry of the detector and encodes the $60^{\circ}$ angle between the detector's arms, and $\theta_{S}$ and $\phi_{S}(t)=\phi_{S 0}+\omega t$ are the latitude and longitude of the source in the detector's coordinate frame, with $\omega \approx 2 \times 10^{-5} \mathrm{rad} / \mathrm{s}$ being the angular frequency of the TianQin satellites. The transforma- tion from the ecliptic coordinates $(\beta, \lambda)$ to the detector coordinates $\left(\theta_{S}, \phi_{S}\right)$ can be found in Appendix E. The subscripts 1 and 2 in Eqs. (7)-(10) are labels for the two Michelson signals, which are orthogonal to each other, as indicated by the $\pi / 4$ phase difference between the corresponding antenna pattern functions [e.g., 91]. From 


\begin{tabular}{l|c}
\hline Configuration & TianQin \\
\hline Number of satellites & $\mathrm{N}=3$ \\
Orientation & $\lambda=120.4^{\circ}, \beta=-4.7^{\circ}$ \\
Observation windows & $2 \times 3$ months each year \\
Mission lifetime & 5 years \\
Arm length & $L=\sqrt{3} \times 10^{5} \mathrm{~km}^{-24} \mathrm{~m}^{2} \mathrm{~Hz}^{-1}$ \\
Displacement measurement noise & $S_{x}=1 \times 10^{-30} \mathrm{~m}^{2} \mathrm{~s}^{-4} \mathrm{~Hz}^{-1}$ \\
\hline Acceleration noise & $S_{a}=1 \times 10^{-3}$
\end{tabular}

TABLE I. Key parameters for the TianQin configurations.

Eqs. (7)-(10) one can conclude that TianQin is most sensitive to GWs propagating along the normal direction to the detector's plane, and least sensitive to GWs propagating along the detector plane.

In general, the exact inclusion of the antenna pattern functions is complicated [e.g., 92]. In practice, we introduce the sky-averaged response function $R(f)$ to simplify the following calculations. It can be approximated by

$$
R(f) \approx \frac{3}{10} \frac{1}{1+0.6\left(f / f_{*}\right)^{2}} .
$$

The prefactor $3 / 10=2 \times 3 / 20$ is two times (to account for two independent Michelson interferometers) the sky-

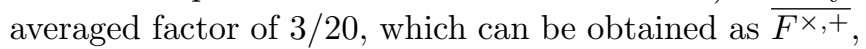
with $\overline{F^{\times,+}} \equiv \frac{1}{4 \pi^{2}} \int_{0}^{\pi} \mathrm{d} \psi_{S} \int_{0}^{2 \pi} \mathrm{d} \phi \int_{0}^{\pi} F^{\times,+} \sin \theta \mathrm{d} \theta$.

\section{Detector noise and the scaled sensitivity curve}

The huge number of Galactic DWDs can generate a foreground confusion noise that may affect the detection of other types of GW sources. In Sect. IV A we show that such foreground is relatively weak for TianQin. Therefore, through this paper we only consider the instrumental noise.

The noise spectral density of TianQin can be expressed analytically as

$$
S_{N}(f)=\frac{1}{L^{2}}\left[\frac{4 S_{a}}{(2 \pi f)^{4}}\left(1+\frac{10^{-4} \mathrm{~Hz}}{f}\right)+S_{x}\right],
$$

where $L, S_{a}, S_{x}$ are given in Table I.

From the sky-averaged response function Eq. (11) and the detector noise Eq. (12), one can construct the sensitivity curve of the detector as

$$
\begin{aligned}
\tilde{S}_{n}(f)= & S_{N}(f) / \tilde{R}(f) \\
= & \frac{1}{L^{2}}\left[\frac{4 S_{a}}{(2 \pi f)^{4}}\left(1+\frac{10^{-4} H z}{f}\right)+S_{x}\right] \\
& \times\left[1+0.6\left(\frac{f}{f_{*}}\right)^{2}\right],
\end{aligned}
$$

where

$$
\tilde{R}(f) \equiv R(f) \frac{10}{3}=\frac{1}{1+0.6\left(f / f_{*}\right)^{2}} .
$$

Note that in this formalism, we assume the effect of the antenna pattern to be associated with the signal. The obtained sensitivity curve is represented in FIG. 2.

\section{Data analysis}

The SNR $\rho$ of a signal is defined as

$$
\rho^{2}=(h \mid h),
$$

where the inner product $(\cdot \mid \cdot)$ is defined as [94, 95],

$$
\begin{aligned}
(a \mid b) & =4 \Re e \int_{0}^{\infty} \mathrm{d} f \frac{\tilde{a}^{*}(f) \tilde{b}(f)}{\tilde{S}_{n}(f)} \\
& \simeq \frac{2}{\tilde{S}_{n}\left(f_{0}\right)} \int_{0}^{T} \mathrm{~d} t a(t) b(t),
\end{aligned}
$$

where $\tilde{a}(f)$ and $\tilde{b}(f)$ are the Fourier transformations of two generic functions $a(t)$ and $b(t), \tilde{S}_{n}(f)$ is defined in Eq. (13). The second step is obtained by using Parseval's theorem and the quasi-monochromatic nature of the signal, which acts like a Dirac delta function on the noise power spectral density [91].

For a monochromatic GW signal with frequency $f_{0}$, it is possible to derive an analytical expression of the SNR $(\rho)^{1}$

$$
\rho^{2}=(h \mid h) \simeq \frac{2}{\tilde{S}_{n}\left(f_{0}\right)} \int_{0}^{T} \mathrm{~d} t h(t) h(t)=\frac{2\left\langle A^{2}\right\rangle T}{\tilde{S}_{n}\left(f_{0}\right)},
$$

with

$$
\left\langle A^{2}\right\rangle=\frac{1}{T} \int_{0}^{T} h^{2}(t) \mathrm{d} t
$$

\footnotetext{
1 Note that our definitions of the SNR and the amplitude differ from those in [23] by a numerical factor, but we both are selfconsistent.
} 


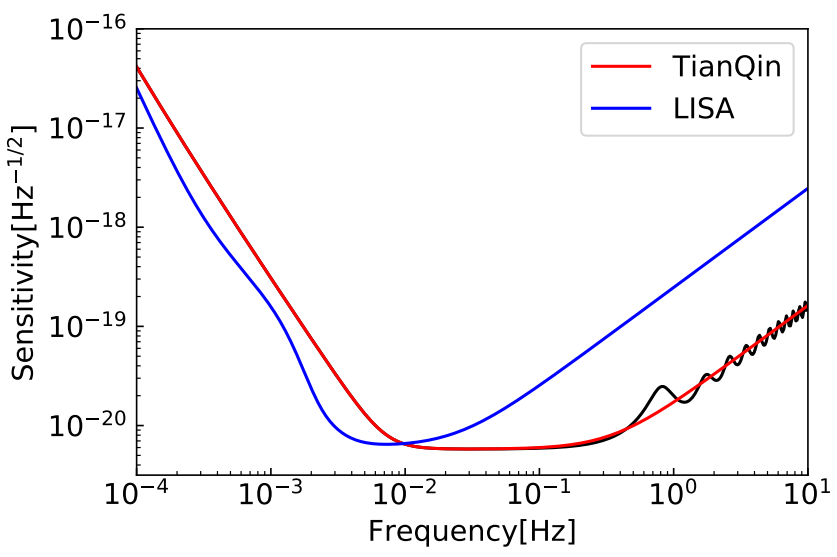

FIG. 2. The sensitivity curve of TianQin. The red line corresponds to $\tilde{S}_{n}(f)$, defined in Eq. (13), while the black line corresponds to the full sky-averaged result, preserving all the frequency dependence (see Eqs. (15)-(16) in [15]). The LISA sensitivity curve is shown by the blue line according to [93].

$$
\begin{aligned}
\approx & \frac{3}{16} \mathcal{A}^{2}\left[\left(1+\cos ^{2} \iota\right)^{2}\left\langle F_{+}^{2}\right\rangle\right. \\
& \left.+4 \cos ^{2} \iota\left\langle F_{\times}^{2}\right\rangle\right], \\
\left\langle F_{+}^{2}\right\rangle= & \frac{1}{4}\left(1+\cos ^{2} \theta_{S}\right)^{2} \cos ^{2} 2 \psi_{S} \\
& +\cos ^{2} \theta_{S} \sin ^{2} 2 \psi_{S}, \\
\left\langle F_{\times}^{2}\right\rangle= & \frac{1}{4}\left(1+\cos ^{2} \theta_{S}\right)^{2} \sin ^{2} 2 \psi_{S} \\
& +\cos ^{2} \theta_{S} \cos ^{2} 2 \psi_{S},
\end{aligned}
$$

where $T$ is the observation time (which is half the operation time), and we have neglected the $\mathcal{O}\left(T^{-1}\right)$ terms in Eq. (19). It is also useful to define the characteristic strain $h_{c}=A \sqrt{N}$, with $N=f_{0} T$ being the number of binary orbital cycles observed during the mission. Analogously, the noise characteristic strain is $h_{n}(f)=$ $\sqrt{f \tilde{S}_{n}(f)}$. One can straightforwardly estimate the SNR from the ratio between $h_{c}$ and $h_{n}$.

\section{E. Galactic GW foreground}

At frequencies $<1 \mathrm{mHz}$, the number of Galactic sources per frequency is too large to resolve all individual GW signals. These signals can potentially become indistinguishable and form a foreground for the TianQin mission [in analogy with 96]. We assess the level of such a foreground using a synthetic population presented in Section II B.

We follow the method outlined in Littenberg and Cornish [97]. For each binary, we construct the signal in the frequency domain, $h(f)$ (cf. Section III A). All signals in each frequency bin are then incoherently added, forming an overall population spectrum. Next, we smooth the spectrum by a running median smoothing function
TABLE II. The coefficients for the polynomial fit for the foreground, as $10^{\sum_{i} a_{i} x^{i}}$, where $x=\log \left(f / 10^{-3}\right)$. Successive rows correspond to increasing operation time $T$.

\begin{tabular}{cccccccc}
\hline$T$ & $a_{0}$ & $a_{1}$ & $a_{2}$ & $a_{3}$ & $a_{4}$ & $a_{5}$ & $a_{6}$ \\
\hline \hline $0.5 \mathrm{yr}$ & -18.6 & -1.22 & 0.009 & -1.87 & 0.65 & 3.6 & -4.6 \\
\hline $1 \mathrm{yr}$ & -18.6 & -1.13 & -0.945 & -1.02 & 4.05 & -4.5 & -0.5 \\
\hline $2 \mathrm{yr}$ & -18.6 & -1.45 & 0.315 & -1.19 & -4.48 & 10.8 & -9.4 \\
\hline $4 \mathrm{yr}$ & -18.6 & -1.43 & -0.687 & 0.24 & -0.15 & -1.8 & -3.2 \\
\hline $5 \mathrm{yr}$ & -18.6 & -1.51 & -0.710 & -1.13 & -0.83 & 13.2 & -19.1 \\
\hline
\end{tabular}

with a set window size and by fitting with cubic spline to it. We define the smoothed Galactic spectrum $S_{\mathrm{DWD}}(f)$ and compute the total noise as the sum of the instrumental noise $S_{n}(f)$ and $S_{\text {DWD }}(f)$. Using the updated noise curve, we check if any DWDs results have a SNR larger than the preset threshold of 7 . These "resolved" DWDs are then removed from the sample, and the process is repeated from the beginning. The iterations are performed until the convergence i.e., until there are no more new resolved sources. The final result is represented in FIG. 3.

\section{F. Parameter estimation}

The uncertainty on the binary parameters can be derived from the Fisher information matrix (FIM) $\Gamma_{i j}$,

$$
\Gamma_{i j}=\left(\frac{\partial h}{\partial \xi_{i}} \mid \frac{\partial h}{\partial \xi_{j}}\right),
$$

where $\xi_{i}$ stands for the $i$ th parameter.

In the high-SNR limit $(\rho \gg 1)$, the inverse of the FIM equals to the variance-covariance matrix, $\Sigma=\Gamma^{-1}$. The 
diagonal entries $\Sigma_{i i}$ give the variances (or mean square errors) of each parameter, $\left(\Delta \xi_{i}\right)^{2}$, while the off-diagonal entries describe the covariances. In numerical calculations, we approximate $\partial h / \partial \xi_{i}$ with numerical differentiation

$$
\frac{\partial h}{\partial \xi_{i}} \approx \frac{\delta h}{\delta \xi_{i}} \equiv \frac{h\left(t, \xi_{i}+\delta \xi_{i}\right)-h\left(t, \xi_{i}-\delta \xi_{i}\right)}{2 \delta \xi_{i}} .
$$

The differentiation steps $\delta \xi_{i}$ were chosen to make the numerical calculation stable [98].

Notice that compared with the uncertainty of each coordinate, we are more interested in the sky localization, which is a combination of the uncertainties of both coordinates [91]:

$$
\Delta \Omega_{\mathrm{S}}=2 \pi|\sin \beta|\left(\Sigma_{\beta \beta} \Sigma_{\lambda \lambda}-\Sigma_{\beta \lambda}^{2}\right)^{1 / 2} .
$$

When a network of independent detectors is considered, the total SNR and FIM of a source can be calculated as

$$
\begin{aligned}
\rho_{\text {total }}^{2} & =\sum_{a} \rho_{a}^{2}=\sum_{a}\left(h_{a} \mid h_{a}\right) \\
\Gamma_{\text {total }} & =\sum_{a} \Gamma_{a}=\sum_{a}\left(\frac{\partial h_{a}}{\partial \xi_{i}} \mid \frac{\partial h_{a}}{\partial \xi_{j}}\right),
\end{aligned}
$$

where the subscript $a$ stands for quantities related to the ath detector.

\section{RESULTS}

In this section we report our results for the TianQin mission. We also consider an alternative version of the mission configuration with the same characteristics (cf. Table I), but oriented perpendicularly to the original TianQin's configuration (pointing towards $\lambda=30.4^{\circ}$ and $\beta=0^{\circ}$ ). In the following, we denote the standard TianQin configuration as TQ and the additional one as TQ II. GW observations can be improved if many detectors are working simultaneously in a network (e.g., the LIGO + Virgo network). Therefore, in this work we also explore the possibility of two detectors TQ and TQ II operating simultaneously, both following the "three months on + three months off" observation scheme as a way to fill the data gaps of each other. We refer to the configuration consisting of the two detectors as TQ I+II. In addition, we also explore the possibility of TQ and TQ I+II operating together with LISA.

\section{A. Galactic foreground}

First, we assess the impact of the Galactic confusion foreground for TianQin. In FIG. 3, we show the estimates of the foreground levels corresponding to different operation times (colored lines) obtained according to the procedure described in Section IV A. Each line can be reproduced by using the expression $S_{\mathrm{DWD}}(f)=10^{\sum_{i} a_{i} x^{i}}$, where $x=\log \left(f / 10^{-3}\right)$ and polynomial coefficients $a_{i}$ are reported in Table II for different operation times.

From FIG. 3, it is evident that the foreground strain is inversely proportional to the operation time. Therefore we did not include the Galactic foreground in the following analysis. Notice that the foreground of TianQin and TianQin II are quite consistent, so change in orientation has relatively minor effect on the overall foreground. This is illustrated in FIG. 3 where foreground of TQ II for 5 year operation time is shown.

\section{B. Verification binaries}

Out of 81 considered candidates (cf. Table V) we find 12 verification binaries with $\mathrm{SNR} \geq 5$ : J0806, V407 Vul, ES Cet, AM CVn, SDSS J1908, HP Lib, CR Boo, V803 Cen, ZTF J1539, SDSS J0651, SDSS J0935, and SDSS J2322, with J0806 having the highest SNR. In particular, we find that J0806 reaches a SNR threshold of 5 already after only two days of observation. We predict that its SNR will reach 36.8 after three months of observation, and will exceed 100 after nominal five years of mission (effectively corresponding to 2.5 years of observation time). In addition, we find three potential verification binaries with $3 \leq \mathrm{SNR}<5$ : SDSS J1351, CXOGBS J1751 and PTF J0533. Figure 4 shows the evolution of the SNR with time for all verification binaries in blue for TianQin (TQ).

In Table VI, we report dimensionless amplitudes $(\mathcal{A})$ and SNRs for all 81 CVBs considering mission configurations: TianQin (TQ), TQ II, and TQ I+II, assuming five years of mission lifetime and setting $\phi_{0}=\pi$ and $\psi_{S}=\pi / 2$ for all binaries. We note that the sky position, orbital inclination and GW frequency of the binary affect SNR by a factor of a few (cf. Eqs.(17)-(21)). For example, V803 Cen and SDSS J0651 have comparable GW amplitudes $\left(16.0 \times 10^{-23}\right.$ and $16.2 \times 10^{-23}$, respectively), but their SNRs differ significantly (6.2 and 26.5, respectively). This difference arises both from the fact that SDSS J0651 is located in a more favorable position in the sky for TianQin (TQ), and the fact that it has a higher frequency than V803 Cen. We also note that because TianQin (TQ) is oriented directly towards J0806, its SNR is the largest across the sample, although its amplitude is not the highest. When considering the TQ II configuration with a different orientation, its SNR decreases by a factor of $\sim 3$.

We find that TQ II can detect 13 verification binaries with $\mathrm{SNR}>5$ and one potential verification binary with $3<\mathrm{SNR}<5$. Being orthogonal to TianQin (TQ), the TQ II configuration is more disadvantageous than for the detection of J0806. However, even with TQ II, J0806 can be detected with a SNR of 41.6. This is because J0806 has the highest frequency across the sample (cf. Table V). With a frequency of $6.22 \mathrm{mHz}$, it is 


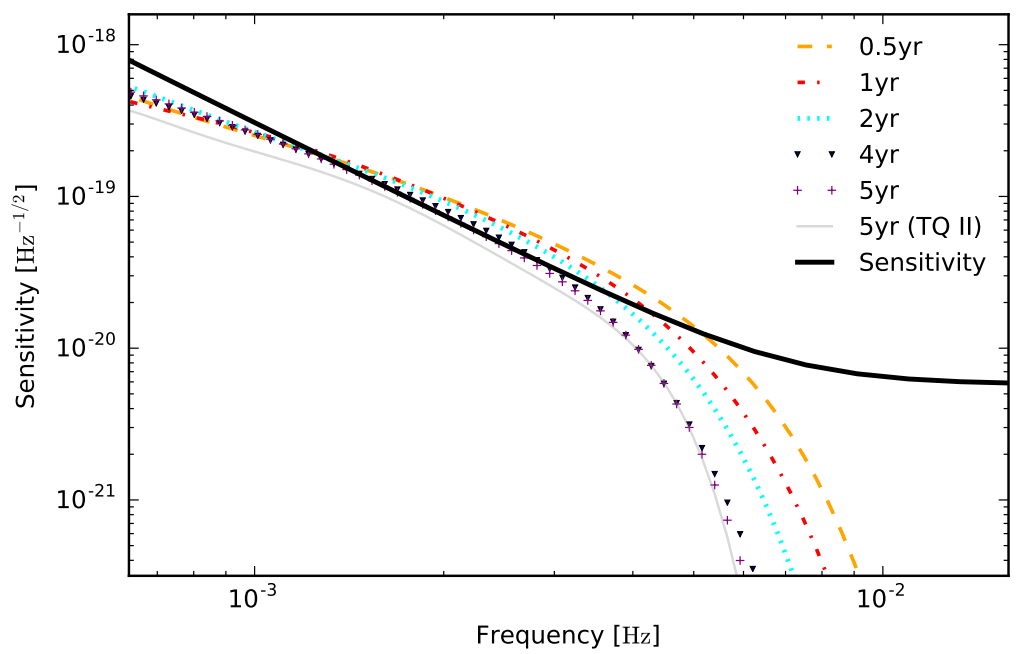

FIG. 3. Expected foreground from Galactic DWDs for different operation times (colored lines). The black solid line represents the sensitivity curve of TianQin (cf. FIG. 2). The foreground with a 5 year operation time for TianQin II is also shown for comparison. Notice that it is consistent with the foreground of the TianQin constellation with the same operation time.

positioned in the amplitude-frequency parameter space where the noise level of TianQin is the lowest (see also FIG. 5). Therefore, J0806 is still among the best verification sources for the TQ II configuration.

Similarly, for the network TQ I+II there will be 14 verification binaries and 1 potential verification binary. The SNR evolution for different operation times for these verification binaries is represented in red in FIG. 4. The SNR produced by a source in this case is given by the root sum squared of the SNRs of the two configurations considered independently (see Eq.(25)). Therefore, if TianQin (TQ) and TQ II independently detect a source with a similar SNR, the network TQ I+II would improve the SNR by a factor of $\sqrt{2}$. However, if the source produces significantly higher SNR in one of the detectors in the network, the improvement is not significant (e.g., J0806 in Table VI).

In Table III, we fix all other parameters and only report the estimated uncertainties on the amplitude $\mathcal{A}$ and inclination angle $\iota$ for the 14 verification binaries. These two parameters are typically degenerate (cf. Eqs. (2)(3)). However, for nearly edge-on binaries the degeneracy can be broken by using the asymmetry between two GW polarizations [e.g. 98]. We present TianQin's ability to constrain the polarization angle $\psi$ in later sections. We remark that [61] demonstrate that in the most favorable setup, TianQin has the potential to constrain the relative strength of extra polarization modes to the tensor modes at an accuracy of about $10^{-3}$. This is reflected in a small correlation coefficient $c_{\mathcal{A}, \cos \iota}=0.157$ of SDSS J0651 with the inclination angle of $86.95^{\circ}$. For decreasing inclination angles the degeneracy increases as can be seen for SDSS J1908 and V803 Cen with inclination angles of $\iota=15^{\circ}$ and $\iota=13.5^{\circ}$ respectively. These two verification binaries have $\Delta \cos \iota>1$, meaning that the uncertainty on the inclination angle exceeds the physical range $(0, \pi)$.

By means of like eclipsing binaries observations, the inclination angle can be independently determined from the EM channel. It can then be used to narrow down the uncertainty on the inclination from GW data by removing the respective row and column of the FIM. In the column denoted "With EM on $\iota$ " in Table III, we recalculate the uncertainties on the amplitude by inverting $\Gamma_{\mathcal{A A}}$, equivalently assuming that the inclination of the binary is known by EM observation, and we report the ratio between this uncertainty and the uncertainty estimated without EM observation on $\iota$ (fourth column of Table III). We find that, when the inclination angle is known a priori, the uncertainty on the amplitude can be improved up by to a factor of 16 (e.g., for SDSS J2322), depending on the exact value of the inclination angle of the source. Note that the improvement for nearly edgeon binaries (ZTF J1539 and SDSS J0651) is negligible.

\section{Simulated Galactic double white dwarf binaries}

To forecast the total number of binaries detectable by TianQin we employ the simulated population of Galactic DWDs (cf. Sec. II B). Here, we set a higher SNR threshold of 7 , assuming that there is no a priori information from the EM observations to fall back on.

We estimate the number of resolved DWDs for the three considered configurations (TQ, TQ II, and TQ I+II) to be of the order of several thousand for the full mission lifetime of 5 years. In Table IV, we summarize our result for increasing operation times. In FIG. 5 we show the dimensionless characteristic strain of DWDs with SNR> 40 in the mock population compared to 14 


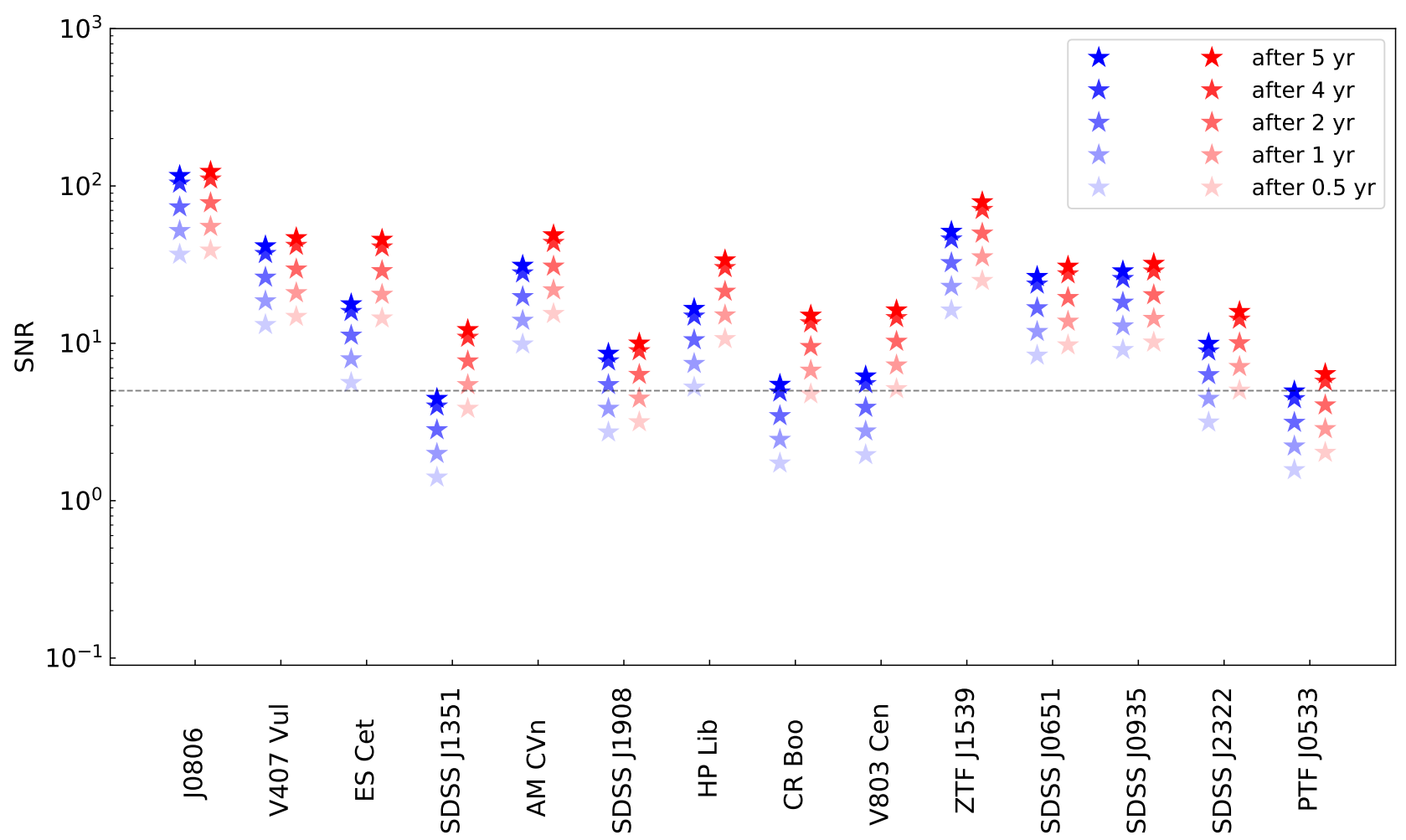

FIG. 4. The SNR evolution of verification binaries over time. Blue stars represent TianQin (TQ), and red stars represent TQ I+II. The black dashed line corresponds to the SNR threshold of 5 .

verification binaries.

The density of DWDs in the bulge region of the Galaxy is significantly higher than in the disk (see Fig. 3 of Korol et al. [52]); therefore the detector's orientation has a significant impact on the total number of detectable DWDs. The Galactic Center (where the density of DWDs is the highest) in ecliptic coordinates corresponds to $\left(\lambda=266.8^{\circ}, \beta=-5.6^{\circ}\right)$. TianQin (TQ) is oriented towards $\left(\lambda=120.4^{\circ}, \beta=-4.7^{\circ}\right)$ that is, about $30^{\circ}$ away from the Galactic Center; TQ II is oriented towards $\left(\lambda=30.4^{\circ}, \beta=0^{\circ}\right)$, which is about $60^{\circ}$ away from the Galactic Center. Consequently, the number of detected DWDs for TianQin (TQ) is about 1.3-1.4 times larger than for TQ II (cf. Table IV). When we consider TQ $\mathrm{I}+\mathrm{II}$, the number of detections increases by $\sim 1.3 \mathrm{com}-$ pared to TianQin (TQ) alone. We verify that pointing the detector towards the Galactic Center would return the maximum detections $\sim 1.0 \times 10^{4}$.

FIG. 6 illustrates the distributions of the SNRs and relative uncertainties on binary parameters $\mathcal{A}, P, \cos \iota$, $\psi_{S}$, and sky position $\Omega_{\mathrm{S}}$ (Eq. 24). The figure shows that most sources have a relatively low SNR $(\leq 10)$, and that there is a non-negligible number of sources with SNR $>100$ reaching a maximum of $\sim 1000$. These high-SNR binaries are also well-localized ones (because $\left.\Delta \Omega_{\mathrm{S}} \propto 1 / \rho^{2}\right)$; theretofore, they will be good candidates for EM follow-up and multi-messenger studies [99]. We find that for $90 \%$ of detections, the uncertainty on $\Delta P / P$ falls within the range $(0.15-4.63) \times 10^{-7}$, on $\Delta \mathcal{A} / \mathcal{A}$ within $0.04-5.02$, on $\Delta \cos \iota$ within $0.02-4.95$, on $\Delta \psi_{S}$ within $0.03-4.01 \mathrm{rad}$, and on $\Delta \Omega_{\mathrm{S}}$ within $0.02-21.36$ $\operatorname{deg}^{2}$. The median values of these uncertainties are: $\Delta P / P=1.41 \times 10^{-7}, \Delta \mathcal{A} / \mathcal{A}=0.26, \Delta \cos \iota=0.20$, $\Delta \psi_{S}=0.39 \mathrm{rad}$, and $\Delta \Omega_{\mathrm{S}}=1.85 \mathrm{deg}^{2}$. We highlight that TianQin (TQ) can locate $39 \%$ of DWDs to within better than $1 \mathrm{deg}^{2}$, while TQ I+II can locate $54 \%$ of detections within $1 \operatorname{deg}^{2}$.

Next, we explore the additional cases of TianQin operating in combination with LISA $^{2}$ : TQ + LISA and TQ I+II + LISA. For these additional cases, the mission lifetimes of TQ and TQ I+II are assumed to be 5 years, while that for LISA is taken to be 4 years [12]. We verify that by adding LISA to the network, the total number of detected DWDs doubles. This is due to the fact that LISA is sensitive to relatively lower $\mathrm{GW}$ frequencies, where the number of DWDs is larger.

As shown in Eq. (25), an additional detector can increase the SNR of a source, and parameter estimation

\footnotetext{
${ }^{2}$ For LISA, we adopt the sensitivity curve from [93].
} 


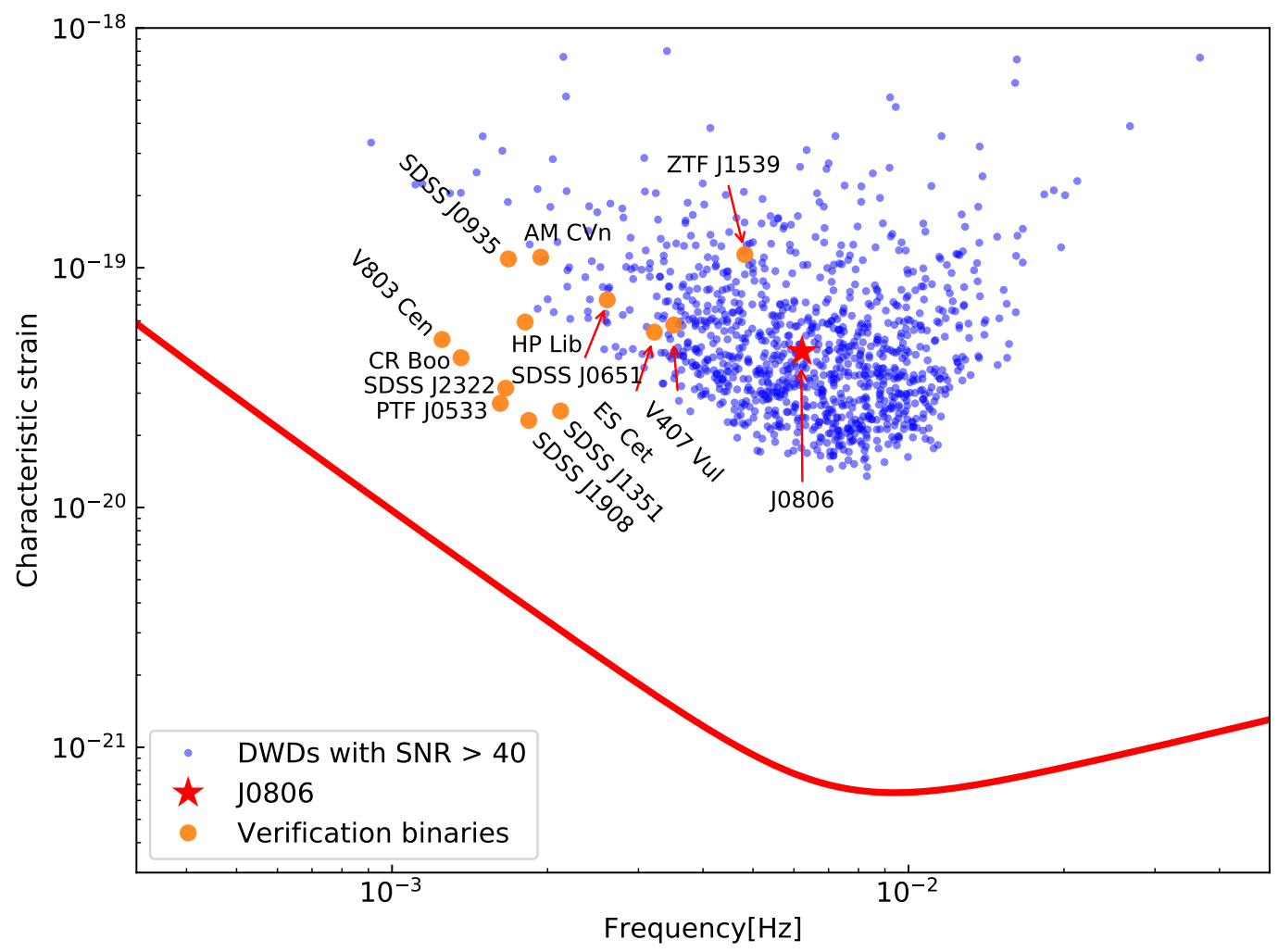

FIG. 5. The characteristic strain $h_{c}$ of the 14 verification binaries (the golden dots and the red star) for TQ I+II and the simulated DWDs with SNR > 40 for TianQin, compared with the noise amplitude $h_{n}$ of TianQin (red line). J0806 is highlighted with a red star. An operation time of 5 years is assumed.

can also benefit. We also look at the improvement in the parameter estimation precision for the 8710 resolvable binaries for TianQin. In FIG. 7, we present the histograms of the ratio between the uncertainties when measured by TianQin alone, and when measured by a network of detectors. The top left panel of FIG. 7 shows that the improvement on the SNR is within a factor of 10 , while the improvements on the parameters uncertainties are within a factor of a few dozens for $\cos \iota$ and $\mathcal{A}$ and are largely comparable for all three networks. Improvements on the SNR, $\mathcal{A}, P, \psi_{S}$, and $\Omega_{\mathrm{S}}$ are larger for TQ + LISA and TQ I+II + LISA; those on $\psi_{S}$ and $\Omega_{\mathrm{S}}$ can reach up to 2 or 3 orders of magnitude.

We remark that (1) TQ + LISA and TQ I+II + LISA are better than TianQin and TQ I+II in determining DWDs' periods. (2) TianQin and TQ I+II are slightly better than TQ + LISA and TQ I+II + LISA in determining GW amplitudes and $\cos (\iota)$. (3) TQ I+II is better than TQ + LISA and TQ I+II + LISA, and the latter two are better than TianQin in determining the sky positions. (4) The result for the polarization angle $\psi_{S}$ is a bit mixed, but the three networks of detectors usually perform better than TianQin alone.

\section{The estimation of the merger rate}

In this section, we estimate the number of DWD mergers that can be expected for TianQin. DWDs typically merge in the frequency ranging from decihertz to a few hertz. Therefore, the inspiral GW signals can be detected by TianQin.

We consider a DWD with equal mass components of $1 \mathrm{M}_{\odot}$, so that the total mass of the binary is larger the Chandrasekhar mass limit. We model its chirping signal with the IMRPhenomPv2 waveform [100] and calculate SNR using Eq. (16). Following Wang et al. [15] and assuming a mission lifetime of 5 years for TianQin, we find that the SNR of our example DWD binary is

$$
\rho \approx 20\left(\frac{1 \mathrm{Mpc}}{d}\right)
$$

This result implies that TianQin can detect SNe Ia explosions within the virial radius of the Local Group.

The SNe Ia rate in the Milky Way is $0.01-0.005 / \mathrm{yr}$ [101], and the DWD merger rate is $4.5-7$ times the SNe Ia rate (as most DWDs would not exceed the Chandrasekhar limit) $[69,102]$. This means that an optimistic estimation of the DWD merger rate is $\sim 0.07 / \mathrm{yr}$ in the Galaxy. 

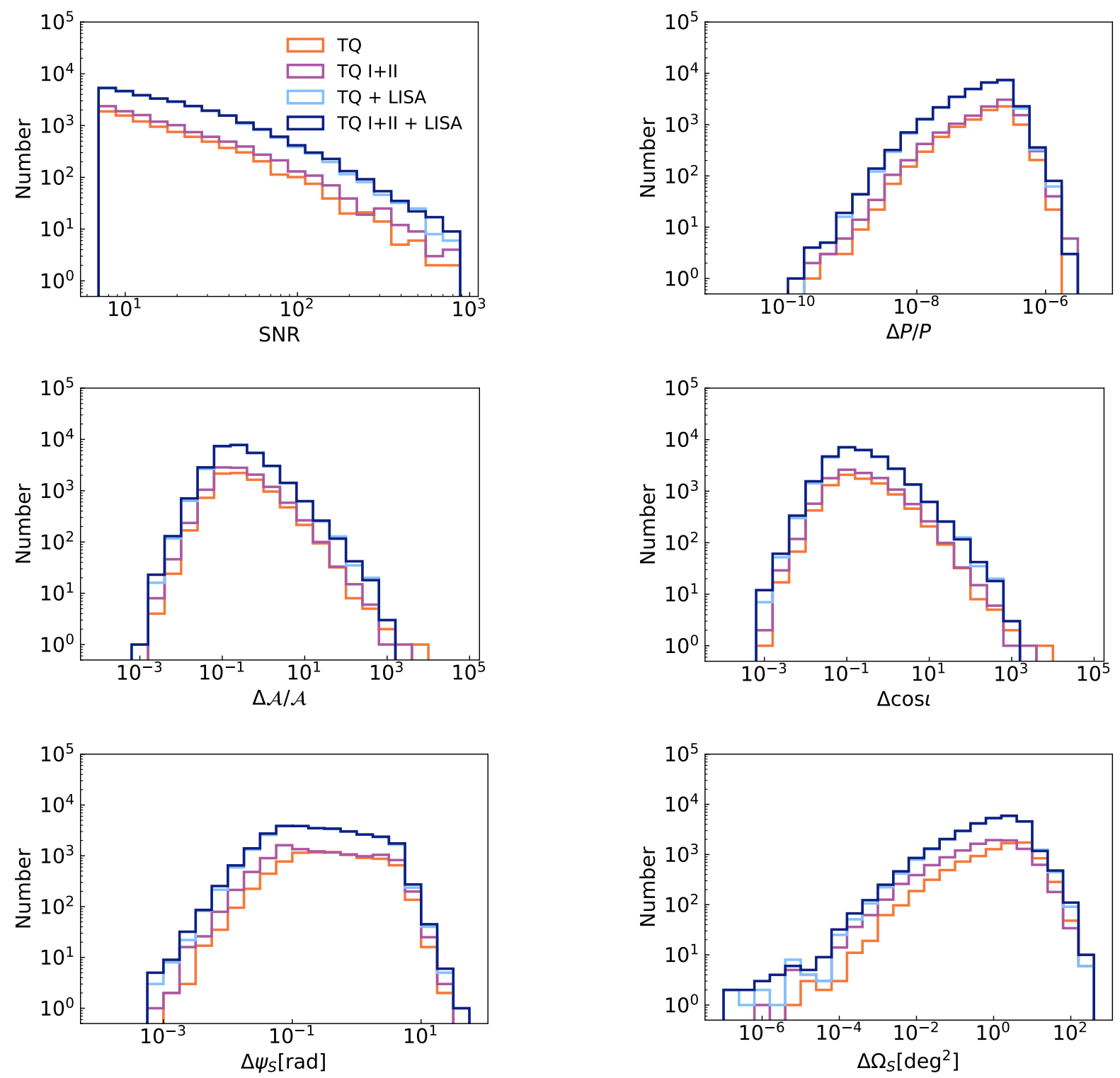

FIG. 6. The histograms (un-normalized) for the SNR and the uncertainties of parameter estimation for the resolvable binaries of different detection scenarios with TianQin (orange), TQ I+II (magenta), TQ + LISA (light blue) and TQ I+II + LISA (blue).

To estimate the DWD merger rate in the Local Group, we note that the Local Group is consists of about 60 galaxies, most with masses $<10^{8} \mathrm{M}_{\odot}$. Therefore, the total mass of the Local Group galaxies is dominated by the Milky Way and the Andromeda Galaxy [103]. The masses of the Milky Way and the Andromeda Galaxy are $0.8-1.5 \times 10^{12} \mathrm{M}_{\odot}$ and $1 \sim 2 \times 10^{12} \mathrm{M}_{\odot}$ [103], respectively. Assuming that the DWD merger rate is proportional to the galaxy mass, one can obtain that the DWD merger rate within the Local Group ranges from $0.0375 / \mathrm{yr}$ to $0.25 / \mathrm{yr}$, using the relation

$$
R_{\mathrm{total}}\left(1+\frac{M_{31}}{M_{\mathrm{MW}}}\right) \times R_{\mathrm{MW}}
$$

where $M_{31}$ and $M_{\mathrm{MW}}$ are masses of Andromeda Galaxy and Milky Way, and $R_{\mathrm{MW}}$ is the DWD merger rate in our Galaxy. Therefore, in the optimistic case, TianQin would be able to observe one DWD merger event with its lifetime of 5 years.

\section{SUMMARY AND DISCUSSION}

In this paper, we carried out the first prediction for the detection of Galactic DWDs with TianQin. For this purpose, we adopted a catalogue of known DWDs discovered with EM observations and a mock Galactic population constructed using a binary population synthesis 

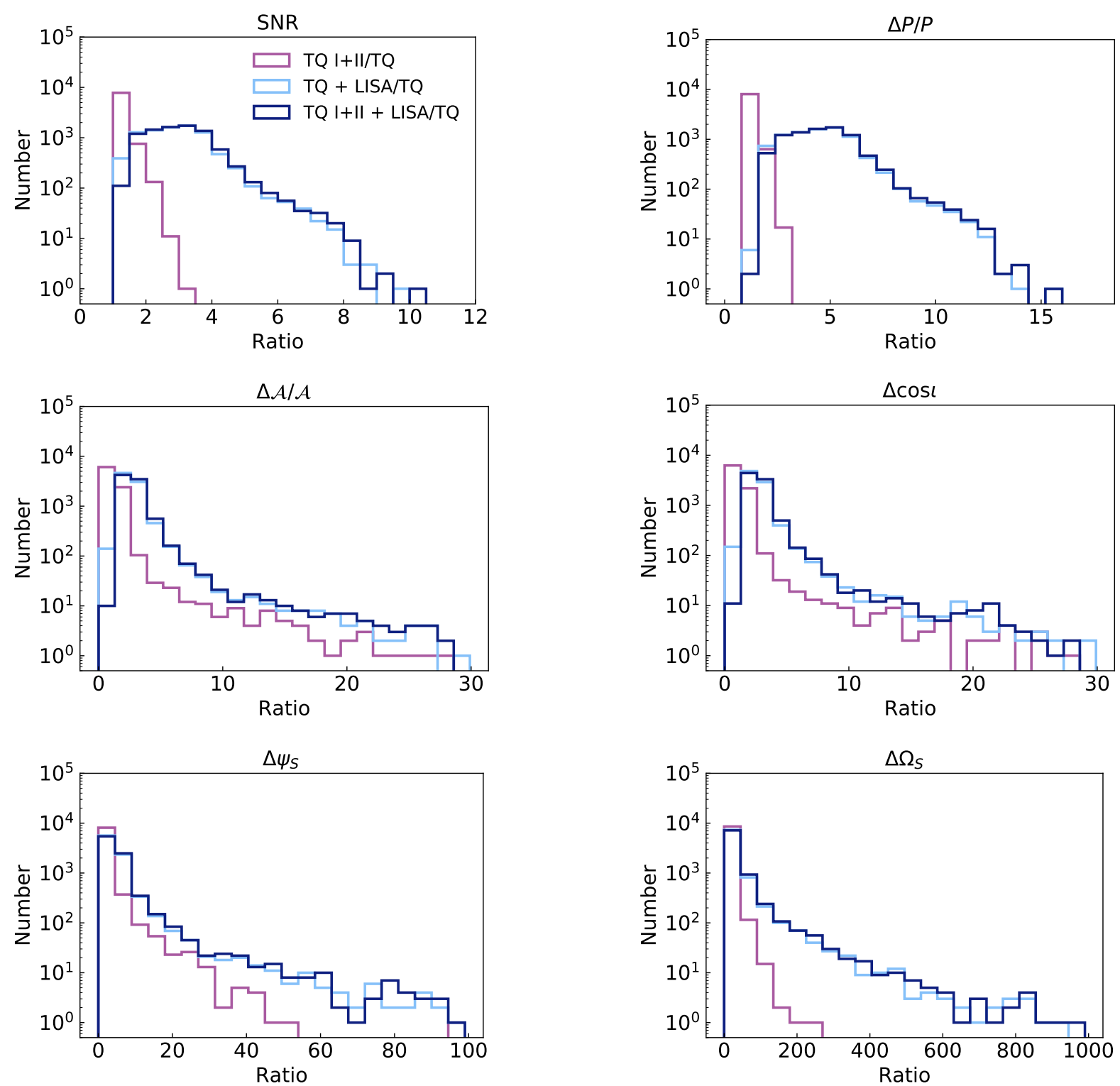

FIG. 7. Histograms showing improvement of parameter estimation uncertainties with repect to TianQin (TQ). The horizontal axis shows the ratio of parameter uncertainties between TQ and the corresponding network, the larger value represents better improvement.

method. We outlined analytical expressions and numerical methods for computing noise curves, SNR, and uncertainties on the measured parameters of monochromatic GW sources for the TianQin mission with fixed orientation. By considering different detector orientations, in this work we also addressed an interesting open question regarding the optimal orientation of the mission.

First, we assessed the strength of the foreground arising from unresolved Galactic DWDs. We found that its effect can be largely ignored for the present design sensitivity of the TianQin detector.

When considering the sample of known DWDs, we found that out of 81 CVBs with orbital periods $\lesssim 5$ hour, TianQin can detect 12 with SNR $\geq 5$ within 5 years of mission lifetime. In particular, we found that TianQin will be able to detect J0806 (its main verification source) already after two days of observations. We estimated that the expected uncertainty on GW amplitude for verification binaries is within a few per cent. For verification binaries with small inclination angles (nearly face-on), this uncertainty can be improved by up to a factor of 16, if the binary inclination angle is known $a$ priori.

When analyzing a synthetic Galactic population of DWD, we found that the overall number of detections is expected to be $8.7 \times 10^{3}$ for the full mission duration of 5 years. We found typical value (median) of $\sim 10^{-7}$ on the relative uncertainty of DWDs' orbital periods, 0.26 
TABLE III. Uncertainties on $\mathcal{A}$ and $\iota$ for 14 verification binaries considering the TQ I+II configuration. In the column denoted "Without EM on $\iota$," we report uncertainties on $\mathcal{A}$ and $\cos \iota$ derived from inverting the $2 \times 2$ FIM. In the column denoted "With EM on $\iota$," we report uncertainties on $\mathcal{A}$ for the case when $\iota$ is known a priori from EM observation.

\begin{tabular}{l|ccc|cc}
\hline \multirow{2}{*}{ Source } & \multicolumn{2}{|c|}{ without EM on $\iota$} & \multicolumn{2}{c}{ with EM on $\iota$} \\
\cline { 2 - 6 } & $\Delta \mathcal{A} / \mathcal{A}$ & $\Delta \cos \iota$ & $c_{\mathcal{A}, \cos \iota}$ & $\Delta^{\prime} \mathcal{A} / \mathcal{A}$ & $\Delta \mathcal{A} / \Delta^{\prime} \mathcal{A}$ \\
\hline \hline J0806 & 0.061 & 0.055 & 0.991 & 0.008 & 7.625 \\
V407 Vul & 0.050 & 0.039 & 0.904 & 0.021 & 2.381 \\
ES Cet & 0.051 & 0.039 & 0.904 & 0.022 & 2.318 \\
SDSS J1351 & 0.193 & 0.145 & 0.905 & 0.082 & 2.354 \\
AM CVn & 0.115 & 0.102 & 0.984 & 0.020 & 5.750 \\
SDSS J1908 & 6.102 & $>1$ & 1.000 & 0.100 & $>1$ \\
HP Lib & 0.384 & 0.360 & 0.997 & 0.030 & 12.800 \\
CR Boo & 0.865 & 0.813 & 0.997 & 0.066 & 13.106 \\
V 803 Cen & 4.377 & $>1$ & 1.000 & 0.062 & $>1$ \\
ZTF J1539 & 0.013 & 0.012 & 0.300 & 0.013 & 1.000 \\
SDSS J0651 & 0.033 & 0.018 & 0.157 & 0.032 & 1.031 \\
SDSS J0935 & 0.073 & 0.056 & 0.904 & 0.031 & 2.355 \\
SDSS J2322 & 1.033 & 0.979 & 0.998 & 0.063 & 16.397 \\
PTF J0533 & 0.219 & 0.137 & 0.700 & 0.156 & 1.404 \\
\hline
\end{tabular}

\begin{tabular}{lcccccc}
\hline & $0.5 y r$ & $1 y r$ & $2 y r$ & $4 y r$ & $5 y r$ \\
\hline \hline TQ & 2371 & 3589 & 5292 & 7735 & 8710 \\
\hline TQ II & 1672 & 2595 & 3943 & 5782 & 6540 \\
\hline
\end{tabular}

TQ I+II 3146471669661002311212

TABLE IV. The expected detection numbers of resolvable binaries for TianQin (TQ), TQ II, and TQ I+II.

on the relative uncertainty of GW amplitude, 0.20 uncertainty on $\cos \iota$, and $\sim 1 \mathrm{deg}^{2}$ uncertainty on sky positions. About $39 \%$ can be localized to within better than $1 \mathrm{deg}^{2}$.

Finally, we outlined a proof-of-principle calculation showing that TianQin is expected to detect one DWD merger event with a supernovae type Ia-like counterpart during its five years of operation time.

In addition to TianQin's nominal orientation (TQ, pointing towards J0806), we also analyzed a variation of the mission oriented perpendicularly (TQ II), and different networks of simultaneously operational GW detectors TQ I+II, TQ + LISA, and TQ I+II + LISA. Although TQ II and TQ I+II can detect the same set of 12 verification binaries as TianQin (TQ), the total number of detections increases by $\sim 1.3$ when considering the network TQ I+II. In addition, the total number of binaries localized to better than $1 \mathrm{deg}^{2}$ also increases to $54 \%$ of the total detected sample. We find that the major advantage of combining TianQin and LISA, besides increasing the total number of detections, consists in the improvement on binary parameter uncertainties by 1-2 orders of magnitude, while the improvement the sky localization can reach up to 3 orders of magnitude.

We are living in the era of large astronomical surveys with the number of known DWDs increasing every year thanks to surveys like ELM [104] and ZTF [105]. The upcoming LSST [106], GOTO [107], and BlackGem [108] will further enlarge the sample by the time TianQin will fly. We show that the TianQin mission has the potential to push the DWD field in the regime of robust statistical studies by increasing the number of detected DWDs to several thousand. By combining data from GW observatories such as TianQin with those from the aforementioned large optical surveys, we will enable multi-messenger studies and advance our knowledge about these unique binary systems.

\section{ACKNOWLEDGMENTS}

We would like to thank Gijs Nelemans, Yan Wang, Jian-dong Zhang, Xin-Chun Hu, Xiao-Hong $\mathrm{Li}$, and Shuxu $\mathrm{Yi}$ for helpful comments and discussions. This work was supported in part by the National Natural Science Foundation of China (Grants No.11703098, No.91636111, No.11690022, No. 1335012, No.11325522, No.11735001, No. 11847241, No.11947210, No.11673031, No.11690024) and the Guangdong Major Project of Basic and Applied Basic Research (Contract No. 2019B030302001). VK acknowledges support from the Netherlands Research Council NWO (Rubicon Grants No.019.183EN.015).

\section{Appendix A: Table of the selected candidate verification binaries}

All the selected CVBs are listed in Table V, with the ecliptic coordinates $(\lambda, \beta)$; the GW frequency $f=2 / P$, with $P$ being the orbital period of the corresponding binary stars; the luminosity distance $d$; the inclination angle $\iota$ of the source; and the heavier and lighter masses, $M$ and $m$, respectively, of the component stars. In some cases, there is no direct measurement on the masses or the inclination angles, so estimated values are assigned based on the evolutionary stage and the mass ratio of the corresponding system. All such values are 
given within square brackets. We make a conservative choice of $5 \mathrm{kpc}$ for the distance to J0806 [79]. The right column of Table $\mathrm{V}$ uses Roman numerals to denote the sources from which the parameters of the listed sources are taken: (i) [76], (ii) [70], (iii) [23], (iv) [71], (v) [74], (vi) [72], (vii) [73], (viii) [104], (ix) [109], and the references therein.

TABLE V: The sample of candidate verification binaries.

${ }^{a}$ As these systems have no measured parallaxes form Gaia DR2, the distance is estimated by other previously observations.

\begin{tabular}{|c|c|c|c|c|c|c|c|c|}
\hline Source & $\begin{array}{c}\lambda \\
{[\mathrm{deg}]}\end{array}$ & $\begin{array}{c}\beta \\
{[\mathrm{deg}]}\end{array}$ & $\begin{array}{c}f \\
{[\mathrm{mHz}]}\end{array}$ & $\begin{array}{c}d \\
{[\mathrm{kpc}]}\end{array}$ & $\begin{array}{c}M \\
{\left[\mathrm{M}_{\odot}\right]}\end{array}$ & $\begin{array}{c}m \\
{\left[\mathrm{M}_{\odot}\right]}\end{array}$ & $\begin{array}{c}\iota \\
\text { [deg] }\end{array}$ & Refs. \\
\hline \multicolumn{9}{|c|}{ AM CVn type systems } \\
\hline J0806 & 120.4425 & -4.7040 & 6.22 & {$[5]^{a}$} & 0.55 & 0.27 & 38 & $\mathrm{i}$ \\
\hline V407 Vul & 294.9945 & 46.7829 & 3.51 & 1.786 & {$[0.8]$} & {$[0.177]$} & {$[60]$} & $\mathrm{i}$ \\
\hline ES Cet & 24.6120 & -20.3339 & 3.22 & 1.584 & {$[0.8]$} & {$[0.161]$} & {$[60]$} & $\mathrm{i}$ \\
\hline SDSS J135154.46-064309.0 & 208.3879 & 4.4721 & 2.12 & 1.317 & {$[0.8]$} & {$[0.100]$} & {$[60]$} & $\mathrm{i}$ \\
\hline $\mathrm{AM} \mathrm{CVn}$ & 170.3858 & 37.4427 & 1.94 & 0.299 & 0.68 & 0.125 & 43 & $\mathrm{i}$ \\
\hline SDSS J190817.07+394036.4 & 298.2172 & 61.4542 & 1.84 & 1.044 & {$[0.8]$} & {$[0.085]$} & 15 & $\mathrm{i}$ \\
\hline HP Lib & 235.0882 & 4.9597 & 1.81 & 0.276 & 0.645 & 0.068 & 30 & $\mathrm{i}$ \\
\hline PTF1 J191905.19+481506.2 & 309.0023 & 69.0290 & 1.48 & 1.338 & {$[0.8]$} & {$[0.066]$} & {$[60]$} & $\mathrm{i}$ \\
\hline ASASSN-14cc & 303.9576 & -42.8640 & 1.48 & 1.019 & {$[0.6]$} & {$[0.01]$} & {$[60]$} & ii \\
\hline CXOGBS J175107.6-294037 & 268.0614 & -6.2526 & 1.45 & 0.971 & {$[0.8]$} & {$[0.064]$} & [60] & $\mathrm{i}$ \\
\hline CR Boo & 202.2728 & 17.8971 & 1.36 & $0.337^{a}$ & 0.885 & 0.066 & 30 & $\mathrm{i}$ \\
\hline KL Dra & 334.1334 & 78.3217 & 1.33 & 0.956 & 0.76 & 0.057 & {$[60]$} & ii,iii \\
\hline V803 Cen & 216.1673 & -30.3166 & 1.25 & $0.347^{a}$ & 0.975 & 0.084 & 13.5 & $\mathrm{i}$ \\
\hline PTF1 J071912.13+485834.0 & 104.3883 & 26.5213 & 1.24 & 0.861 & {$[0.8]$} & {$[0.053]$} & {$[60]$} & i,ii \\
\hline SDSS J092638.71+3 & 132.2867 & 20.2342 & 1.18 & 0.577 & 0.85 & 0.035 & 82.6 & ii,iii \\
\hline CP Eri & 42.1327 & -26.4276 & 1.17 & & {$[0.8]$} & {$[0.049]$} & {$[60]$} & $\mathrm{i}, \mathrm{ii}$ \\
\hline SDSS J104325. & 136.2923 & 43.9158 & 1.17 & 0.979 & {$[0.6]$} & {$[0.01]$} & {$[60]$} & ii \\
\hline $\mathrm{CF}$ & 147.3411 & -34.5979 & 1.12 & 1.113 & {$[0.6]$} & {$[0.01]$} & {$[60]$} & ii \\
\hline $\mathrm{CR}$ & 22.5049 & 11.1283 & 1.05 & 0.734 & {$[0.6]$} & {$[0.01]$} & {$[60]$} & ii \\
\hline $06 \mathrm{Hy}$ & 140.7336 & -21.2342 & 0.99 & 0.504 & {$[0.8]$} & {$[0.040]$} & {$[60]$} & $\mathrm{i}, \mathrm{ii}$ \\
\hline SDSS J173047.59- & 248.6846 & 78.6529 & 0.95 & 0.911 & {$[0.6]$} & {$[0.01]$} & {$[60]$} & ii \\
\hline 2QZ J142701.6-0 & 214.8878 & 12.4608 & 0.91 & 0.677 & {$[0.6]$} & {$[0.015]$} & [60] & ii,iii \\
\hline SDSS J124058.03- & 190.1933 & 2.2262 & 0.89 & 0.577 & [0.8] & {$[0.035]$} & [60] & i,ii \\
\hline NSV1440 & 283.1788 & -72.6108 & 0.89 & 0.377 & {$[0.6]$} & {$[0.01]$} & {$[60]$} & ii \\
\hline SDSS J012940.05+384210.4 & 35.8760 & 27.0847 & 0.89 & 0.508 & {$[0.8]$} & {$[0.034]$} & [60] & i,ii \\
\hline SDSS J172102.48+273301.2 & 256.3525 & 50.5292 & 0.87 & 0.995 & {$[0.6]$} & {$[0.01]$} & [60] & ii \\
\hline ASASSN-14mv & 107.1184 & -1.4233 & 0.82 & 0.247 & {$[0.6]$} & {$[0.01]$} & {$[60]$} & ii \\
\hline ASASSN & 5.2639 & -59.9095 & 0.78 & 0.255 & {$[0.6]$} & {$[0.01]$} & {$[60]$} & ii \\
\hline (15 & 4.3101 & 52.2364 & 0.75 & 0.524 & {$[0.6]$} & {$[0.01]$} & {$[60]$} & ii \\
\hline & 119.9052 & -3.9847 & 0.75 & 0.828 & [0.8] & {$[0.027]$} & [60] & i,ii \\
\hline SDSS J14111 & 183.5559 & 55.8748 & 0.72 & 0.429 & {$[0.6]$} & {$[0.01]^{\top}$} & {$[60]$} & ii \\
\hline GP & 187.7210 & 23.0012 & 0.72 & 0.073 & 0.59 & 0.011 & {$[60]$} & ii,iii \\
\hline SDSS J09022 & 126.7527 & 20.5254 & 0.69 & 0.461 & {$[0.6]$} & {$[0.01]$} & {$[60]$} & ii \\
\hline ASASS & 183.9170 & 78.0916 & 0.67 & 0.259 & 0.87 & 0.025 & 86.3 & i,ii \\
\hline SDSS J120841 & 165.8193 & 33.3289 & 0.63 & 0.202 & {$[0.8]$} & {$[0.022]$} & {$[60]$} & i,ii \\
\hline SS J164228.C & 245.3756 & 41.3659 & 0.62 & 1.044 & {$[0.6]$} & {$[0.01]$} & [60] & ii \\
\hline SDSS J155252.48+ & 225.2376 & 50.6483 & 0.59 & 0.443 & {$[0.6]$} & [0.01] & [60] & ii \\
\hline SDSS J113732.32+4 & 156.4126 & 34.8546 & 0.56 & 0.209 & {$[0.6]$} & {$[0.01]$} & [60] & ii \\
\hline V396 Hya/CE & 205.7504 & -14.4638 & 0.51 & 0.094 & {$[0.8]$} & {$[0.016]$} & [60] & i,ii \\
\hline SDSS J1319+5915 & 159.2931 & 59.0926 & 0.51 & 0.205 & {$[0.6]$} & {$[0.01]$} & [60] & ii \\
\hline \multicolumn{9}{|c|}{ detached DWD } \\
\hline & 205.0315 & 66.1616 & 4.82 & 1.262 & 0.61 & 0.21 & 84 & iv \\
\hline & & & 2.61 & & 0.247 & 0.49 & 86.9 & $\mathrm{i}$ \\
\hline SDSS J093506.92+441107.0 & 130.9795 & 28.0912 & 1.68 & $0.645^{a}$ & 0.312 & 0.75 & {$[60]$} & $\mathrm{i}$ \\
\hline SDSS J232230.20+050942.06 & 353.4373 & 8.4572 & 1.66 & 0.779 & 0.24 & 0.27 & 27 & $\mathrm{v}$ \\
\hline PTF J053332.05+020911.6 & 82.9097 & -21.1234 & 1.62 & 1.253 & 0.65 & 0.167 & 72.8 & vi \\
\hline SDSS J010657.39-100003.3 & 11.4582 & -15.7928 & 0.85 & 0.758 & 0.188 & 0.57 & 67 & i,iii \\
\hline SDSS J163030.58+423305.7 & 231.7612 & 63.0501 & 0.84 & 1.019 & 0.298 & 0.76 & {$[60]$} & $\mathrm{i}$ \\
\hline SDSS J082239.54+304857.2 & 120.6816 & 11.0965 & 0.83 & 0.861 & 0.304 & 0.524 & 88.1 & i,iii \\
\hline ZTF J190125.42+530929.5 & 306.8131 & 74.6335 & 0.82 & 0.898 & 0.50 & 0.20 & 86.2 & vii \\
\hline SDSS J104336.27+055149.9 & 160.1545 & -2.0480 & 0.73 & 1.744 & 0.183 & 0.76 & {$[60]$} & i,iii \\
\hline
\end{tabular}


TABLE V - Continued from previous page

\begin{tabular}{|c|c|c|c|c|c|c|c|c|}
\hline Source & $\begin{array}{c}\lambda \\
{[\mathrm{deg}]} \\
\end{array}$ & $\begin{array}{c}\beta \\
{[\mathrm{deg}]} \\
\end{array}$ & $\begin{array}{c}f \\
{[\mathrm{mHz}]}\end{array}$ & $\begin{array}{c}d \\
{[\mathrm{kpc}]}\end{array}$ & $\begin{array}{c}M \\
{\left[\mathrm{M}_{\odot}\right]}\end{array}$ & $\left.\begin{array}{c}m \\
{\left[\mathrm{M}_{\odot}\right]}\end{array}\right]$ & $\begin{array}{c}\iota \\
{[\mathrm{deg}]}\end{array}$ & Refs. \\
\hline SDSS J105353.89+520031.0 & 141.2200 & 40.8002 & 0.54 & 0.683 & 0.204 & 0.75 & {$[60]$} & i,iii \\
\hline SDSS J005648.23-061141.5 & 10.6273 & -11.3044 & 0.53 & 0.620 & 0.180 & 0.82 & [60] & $\mathrm{i}, \mathrm{iii}$ \\
\hline SDSS J105611.02+653631.5 & 130.4076 & 52.2268 & 0.53 & 1.104 & 0.334 & 0.76 & {$[60]$} & i,iii \\
\hline SDSS J092345.59+302805.0 & 133.7151 & 14.4268 & 0.51 & 0.299 & 0.275 & 0.76 & [60] & $\mathrm{i}$ \\
\hline SDSS J143633.28+501026.9 & 187.5011 & 59.9313 & 0.50 & 1.011 & 0.234 & 0.78 & [60] & i,iii \\
\hline SDSS J082511.90+115236.4 & 125.7257 & -7.1746 & 0.40 & 1.786 & 0.278 & 0.80 & {$[60]$} & i,iii \\
\hline WD 09 & 208.5263 & -67.3013 & 0.38 & 0.163 & 0.37 & 0.32 & 68 & i,iii \\
\hline SDSS J174140 & 208.8283 & 87.8286 & 0.38 & 1.159 & 0.170 & 1.17 & {$[60]$} & i,iii \\
\hline SDSS J075552 & 110.9953 & 27.7583 & 0.37 & $2.620^{a}$ & 0.176 & 0.81 & {$[60]$} & iii \\
\hline & 46.5446 & -16.9689 & 0.30 & 0.429 & 0.15 & 0.263 & {$[60]$} & iii \\
\hline SDSS J23091؛ & 359.6100 & 28.7808 & 0.30 & 1.765 & 0.176 & 0.96 & {$[60]$} & viii \\
\hline SDSS J084910.13+044528.7 & 133.3917 & -12.5404 & 0.29 & 1.002 & 0.176 & 0.65 & [60] & iii \\
\hline SDSS J002207.65-101423.5 & 0.9548 & -11.5858 & 0.29 & $1.151^{a}$ & 0.21 & 0.375 & [60] & iii \\
\hline SDSS J075141.18-014120.9 & 120.3746 & -22.2324 & 0.29 & 1.741 & 0.97 & 0.194 & [60] & iii \\
\hline SDSS J211921.96-001825.8 & 322.1533 & 14.5725 & 0.27 & 1.053 & 0.74 & 0.158 & [60] & iii \\
\hline SDSS J123410.36-022802.8 & 188.8196 & 1.1194 & 0.25 & 0.754 & 0.09 & 0.23 & {$[60]$} & iii \\
\hline SDSS J100559.10+224932.2 & 145.4432 & 10.4254 & 0.24 & 0.555 & 0.36 & 0.31 & 88.9 & iii \\
\hline SDSS J115219.99+024814.4 & 177.1265 & 1.8106 & 0.23 & 0.718 & 0.47 & 0.41 & 89.2 & iii \\
\hline SDSS J & 8923 & -26.0 & 0.22 & 1.313 & 0.39 & 0.168 & {$[60]$} & iii \\
\hline SDSS J07 & 1.6397 & 3939 & 0.20 & 0.875 & 0.1 & 0.156 & [60] & iii \\
\hline WD 12 & 194.5586 & -5.5520 & 0.19 & 0.040 & 0.56 & 0.39 & 45.1 & i,iii \\
\hline SDSS J110815 & 162.1662 & 8.9070 & 0.19 & $0.698^{a}$ & 0.42 & 0.167 & {$[60]$} & iii \\
\hline WD $1101+36$ & 152.2513 & 27.6895 & 0.16 & 0.088 & 0.36 & 0.31 & {$[60]$} & iii \\
\hline WD $1704+4807 \mathrm{BC}$ & 242.3234 & 70.1865 & 0.16 & 0.039 & 0.39 & 0.56 & {$[60]$} & ix \\
\hline SDSS J011210.25+183503.7 & 23.7268 & 10.1149 & 0.16 & 0.843 & 0.62 & 0.16 & [60] & iii \\
\hline SDSS J123316.20+160204.6 & 181.0654 & 17.9826 & 0.15 & 1.207 & 0.169 & 0.98 & [60] & viii \\
\hline SDSS J113017.42+385549.9 & 156.0760 & 32.4474 & 0.15 & 0.884 & 0.72 & 0.286 & [60] & iii \\
\hline SDSS J111215.82+111745 & 164.6171 & 5.6844 & 0.13 & 0.384 & 0.14 & 0.169 & [60] & iii \\
\hline SDSS J100554.05+3! & 140.4558 & 22.5307 & 0.13 & 1.747 & 0.168 & 0.75 & [60] & viii \\
\hline & 213.1397 & 29.4368 & 0.12 & 0.839 & 0.84 & 0.181 & {$[60]$} & iii \\
\hline SDSS J184037.78+642312.3 & 337.4095 & 85.2636 & 0.12 & 0.829 & 0.65 & 0.177 & {$[60]$} & iii \\
\hline
\end{tabular}

\section{Appendix B: SNR OF CANDIDATE VERIFICATION BINARIES}

The GW amplitudes and SNR of all selected CVBs are listed in Table VI, assuming a nominal mission lifetime of five years and the three configurations of TianQin, $\phi_{0}=\pi$ and $\psi_{S}=\pi / 2$ for all binaries.

TABLE VI: The expected amplitude $\mathcal{A}$ and SNR of 81 candidate verification binaries. $\mathcal{A}$ is given in units of $10^{-23}$.

\begin{tabular}{ccccc}
\hline Source & $\mathcal{A}$ & \multicolumn{3}{c}{ SNR } \\
& \multicolumn{5}{c}{ TQ } & TQ II & TQ I+II \\
\hline \hline AM CVn type systems \\
J0806 & 6.4 & 116.202 & 41.657 & 123.443 \\
V407 Vul & 11.0 & 41.528 & 21.537 & 46.780 \\
ES Cet & 10.7 & 17.775 & 42.110 & 45.708 \\
SDSS J135154.46-064309.0 & 6.2 & 4.454 & 11.345 & 12.188 \\
AM CVn & 28.3 & 31.245 & 37.499 & 48.810 \\
SDSS J190817.07+394036.4 & 6.1 & 8.622 & 5.077 & 10.006 \\
HP Lib & 15.7 & 16.619 & 29.427 & 33.795 \\
PTF1 J191905.19+481506.2 & 3.2 & 1.526 & 1.122 & 1.894 \\
ASASSN-14cc & 0.5 & 0.338 & 0.188 & 0.387 \\
CXOGBS J175107.6-294037 & 4.2 & 3.022 & 2.172 & 3.722 \\
CR Boo & 12.9 & 5.473 & 14.029 & 15.058 \\
\hline & \multicolumn{5}{c}{ Continued on next page }
\end{tabular}


TABLE VI - Continued from previous page

\begin{tabular}{|c|c|c|c|c|}
\hline \multirow[t]{2}{*}{ Source } & \multirow[t]{2}{*}{$\mathcal{A}$} & \multicolumn{3}{|c|}{ SNR } \\
\hline & & TQ & TQ II & TQ I+II \\
\hline KL Dra & 3.5 & 1.109 & 1.006 & 1.497 \\
\hline V803 Cen & 16.0 & 6.187 & 15.026 & 16.249 \\
\hline PTF1 J071912.13+485834.0 & 3.6 & 1.844 & 0.982 & 2.089 \\
\hline SDSS J092638.71+362402.4 & 3.6 & 1.175 & 0.664 & 1.350 \\
\hline CP Eri & 2.8 & 0.676 & 1.384 & 1.540 \\
\hline SDSS J104325.08+563258.1 & 0.5 & 0.178 & 0.112 & 0.211 \\
\hline CRTS J0910-2008 & 0.4 & 0.164 & 0.104 & 0.194 \\
\hline CRTS J0105+1903 & 0.6 & 0.108 & 0.256 & 0.277 \\
\hline V406 Hya/2003aw & 4.0 & 1.414 & 0.745 & 1.599 \\
\hline SDSS J173047.59+554518.5 & 0.4 & 0.069 & 0.066 & 0.096 \\
\hline 2QZ J142701.6-012310 & 0.9 & 0.115 & 0.282 & 0.304 \\
\hline SDSS J124058.03-015919.2 & 2.8 & 0.436 & 0.842 & 0.948 \\
\hline NSV1440 & 1.0 & 0.140 & 0.130 & 0.191 \\
\hline SDSS J012940.05+384210.4 & 3.1 & 0.389 & 0.882 & 0.964 \\
\hline SDSS J172102.48+273301.2 & 0.4 & 0.069 & 0.063 & 0.093 \\
\hline ASASSN- $14 \mathrm{mv}$ & 1.5 & 0.388 & 0.174 & 0.425 \\
\hline ASASSN-14ei & 1.4 & 0.133 & 0.192 & 0.233 \\
\hline SDSS J152509.57+360054.5 & 0.7 & 0.059 & 0.097 & 0.114 \\
\hline SDSS J080449.49+161624.8 & 1.4 & 0.304 & 0.119 & 0.326 \\
\hline SDSS J141118.31+481257.6 & 0.8 & 0.068 & 0.093 & 0.115 \\
\hline GP Com & 5.0 & 0.490 & 0.882 & 1.009 \\
\hline SDSS J090221.35+381941.9 & 0.7 & 0.121 & 0.053 & 0.132 \\
\hline ASASSN-14cn & 4.0 & 0.220 & 0.227 & 0.316 \\
\hline SDSS J120841.96+355025.2 & 4.1 & 0.383 & 0.408 & 0.560 \\
\hline SDSS J164228.06+193410.0 & 0.3 & 0.025 & 0.029 & 0.038 \\
\hline SDSS J155252.48+320150.9 & 0.7 & 0.039 & 0.060 & 0.072 \\
\hline SDSS J113732.32+405458.3 & 1.4 & 0.110 & 0.095 & 0.145 \\
\hline V396 Hya/CE 315 & 5.5 & 0.221 & 0.535 & 0.579 \\
\hline SDSS J1319+5915 & 1.3 & 0.063 & 0.062 & 0.089 \\
\hline \multicolumn{5}{|c|}{ detached DWD } \\
\hline ZTF J153932.16+502738.8 & 18.4 & 51.351 & 60.184 & 79.114 \\
\hline SDSS J065133.34+284423.4 & 16.2 & 26.535 & 15.700 & 30.831 \\
\hline SDSS J093506.92+441107.0 & 29.9 & 28.797 & 14.245 & 32.128 \\
\hline SDSS J232230.20+050942.06 & 8.7 & 9.973 & 12.371 & 15.891 \\
\hline PTF J053332.05+020911.6 & 7.6 & 4.965 & 4.042 & 6.402 \\
\hline SDSS J010657.39-100003.3 & 8.3 & 0.989 & 1.892 & 2.135 \\
\hline SDSS J163030.58+423305.7 & 11.6 & 1.423 & 1.721 & 2.233 \\
\hline SDSS J082239.54+304857.2 & 10.4 & 1.713 & 0.887 & 1.929 \\
\hline ZTF J190125.42+530929.5 & 6.5 & 0.614 & 0.549 & 0.824 \\
\hline SDSS J104336.27+055149.9 & 3.9 & 0.649 & 0.561 & 0.857 \\
\hline SDSS J105353.89+520031.0 & 9.0 & 0.698 & 0.457 & 0.835 \\
\hline SDSS J005648.23-061141.5 & 9.3 & 0.475 & 0.931 & 1.045 \\
\hline SDSS J105611.02+653631.5 & 8.7 & 0.570 & 0.378 & 0.684 \\
\hline SDSS J092345.59+302805.0 & 26.2 & 2.422 & 1.138 & 2.675 \\
\hline SDSS J143633.28+501026.9 & 6.7 & 0.262 & 0.359 & 0.444 \\
\hline SDSS J082511.90+115236.4 & 3.9 & 0.235 & 0.094 & 0.253 \\
\hline WD 0957-666 & 25.7 & 0.502 & 0.621 & 0.798 \\
\hline SDSS J174140.49+652638.7 & 4.9 & 0.104 & 0.103 & 0.147 \\
\hline SDSS J075552.40+490627.9 & 1.7 & 0.072 & 0.035 & 0.080 \\
\hline SDSS J233821.51-205222.8 & 3.3 & 0.073 & 0.078 & 0.107 \\
\hline SDSS J230919.90+260346.7 & 2.4 & 0.046 & 0.062 & 0.077 \\
\hline SDSS J084910.13+044528.7 & 3.2 & 0.094 & 0.042 & 0.103 \\
\hline SDSS J002207.65-101423.5 & 2.1 & 0.036 & 0.056 & 0.067 \\
\hline SDSS J075141.18-014120.9 & 2.7 & 0.078 & 0.032 & 0.084 \\
\hline SDSS J211921.96-001825.8 & 2.9 & 0.069 & 0.037 & 0.078 \\
\hline SDSS J123410.36-022802.8 & 0.9 & 0.011 & 0.020 & 0.022 \\
\hline SDSS J100559.10+224932.2 & 5.3 & 0.059 & 0.039 & 0.071 \\
\hline SDSS J115219.99+024814.4 & 6.3 & 0.047 & 0.062 & 0.078 \\
\hline SDSS J105435.78-212155.9 & 1.3 & 0.014 & 0.017 & 0.022 \\
\hline
\end{tabular}




\begin{tabular}{ccccc}
\multicolumn{5}{c}{ TABLE VI - Continued from previous page } \\
\hline Source & $\mathcal{A}$ & \multicolumn{3}{c}{ SNR } \\
& & TQ & TQ II & TQ I+II \\
\hline \hline SDSS J074511.56+194926.5 & 0.6 & 0.008 & 0.003 & 0.008 \\
WD 1242-105 & 109.2 & 0.755 & 1.656 & 1.820 \\
SDSS J110815.50+151246.6 & 2.4 & 0.022 & 0.020 & 0.030 \\
WD 1101+364 & 25.4 & 0.159 & 0.120 & 0.199 \\
WD 1704+4807BC & 99.9 & 0.376 & 0.388 & 0.541 \\
SDSS J011210.25+183503.7 & 2.2 & 0.008 & 0.019 & 0.020 \\
SDSS J123316.20+160204.6 & 2.2 & 0.009 & 0.014 & 0.016 \\
SDSS J113017.42+385549.9 & 3.9 & 0.019 & 0.016 & 0.026 \\
SDSS J111215.82+111745.0 & 1.4 & 0.005 & 0.005 & 0.008 \\
SDSS J100554.05+355014.2 & 1.1 & 0.005 & 0.003 & 0.006 \\
SDSS J144342.74+150938.6 & 2.6 & 0.005 & 0.010 & 0.011 \\
SDSS J184037.78+642312.3 & 2.1 & 0.004 & 0.004 & 0.005 \\
\hline
\end{tabular}

\section{Appendix C: Re-expression of the responsed Gravitational Wave Signal}

For convenience of calculation, we rearrange the expression for the waveform in the detector:

$$
h(t)=A(t) \cos \Psi(t),
$$

where the waveform amplitude $A(t)$ is

$$
A(t)=\left[\left(A_{+} F^{+}(t)\right)^{2}+\left(A_{\times} F^{\times}(t)\right)^{2}\right]^{1 / 2} .
$$

$A_{+}$and $A_{\times}$are given by

$$
A_{+}=\mathcal{A}\left(1+\cos \iota^{2}\right), \quad A_{\times}=2 \mathcal{A} \cos \iota .
$$

The phase of the waveform is

$$
\Psi(t)=2 \pi f t+\phi_{0}+\Phi_{D}(t)+\Phi_{P}(t)
$$

The polarization phase $\Phi_{P}(t)$ is given by

$$
\Phi_{P}(t)=\tan ^{-1}\left(\frac{-A_{\times} F^{\times}(t)}{A_{+} F^{+}(t)}\right)
$$

\section{Appendix D: Derivation of the average amplitude}

In order to verify our SNR calculation, more specifically the calculation of average amplitude, we can obtain the average amplitude from the antenna beam patterns function given by Eq. (13) in [59]:

$$
\begin{aligned}
& F^{+}(t, \theta, \phi, \psi)=\cos 2 \psi \xi^{+}(t ; \theta, \phi)-\sin 2 \psi \xi^{\times}(t ; \theta, \phi), \\
& F^{\times}(t, \theta, \phi, \psi)=\sin 2 \psi \xi^{+}(t ; \theta, \phi)+\cos 2 \psi \xi^{\times}(t ; \theta, \phi),
\end{aligned}
$$

and

$$
\begin{aligned}
\xi^{+}(t ; \theta, \phi)= & \frac{\sqrt{3}}{32}\left(4 \cos 2\left(\kappa-\beta^{\prime}\right)\left((3+\cos 2 \theta) \sin \theta_{s} \sin 2\left(\phi-\phi_{s}\right)+2 \sin \left(\phi-\phi_{s}\right) \sin 2 \theta \cos \theta_{s}\right)\right. \\
& -\sin 2\left(\kappa-\beta^{\prime}\right)\left(3+\cos 2\left(\phi-\phi_{s}\right)\left(9+\cos 2 \theta\left(3-\cos 2 \theta_{s}\right)\right)+6 \cos 2 \theta_{s} \sin ^{2}\left(\phi-\phi_{s}\right)\right. \\
& \left.\left.-6 \cos 2 \theta \cos ^{2} \theta_{s}+4 \cos \left(\phi-\phi_{s}\right) \sin 2 \theta \sin 2 \theta_{s}\right)\right), \\
\xi^{\times}(t ; \theta, \phi)= & \frac{\sqrt{3}}{8}\left(-4 \cos 2\left(\kappa-\beta^{\prime}\right)\left(\cos 2\left(\phi-\phi_{s}\right) \cos \theta \sin \theta_{s}+\cos \left(\phi-\phi_{s}\right) \sin \theta \cos \theta_{s}\right)\right.
\end{aligned}
$$




$$
\left.+\sin 2\left(\kappa-\beta^{\prime}\right)\left(\cos \theta\left(3-\cos 2 \theta_{s}\right) \sin 2\left(\phi_{s}-\phi\right)+2 \sin \left(\phi_{s}-\phi\right) \sin \theta \sin 2 \theta_{s}\right)\right) .
$$

where $\kappa=2 \pi f_{s c} t+\lambda^{\prime}, f_{s c} \approx 1 /(3.65 d)$ is the modulation frequency from the rotation of the satellites around the guiding center. $\lambda^{\prime}$ and $\beta^{\prime}$ are some of initial phase of constant.

In the above expression, $\theta=\pi / 2-\beta$ and $\phi=\lambda$ are the source location in the ecliptic coordinate system. $\psi$ is the polarization angle. $\theta_{s}$ and $\phi_{s}$ are the ecliptic coordinates of the reference source. For the reference source of TianQin is $\mathrm{J} 0806, \theta_{s}=-4.7040^{\circ}$ and $\phi_{s}=120.4425^{\circ}$.

By performing the same process as described in Section IIID, we get some expressions similar to Eqs. (19)-(21), given below:

$$
\begin{aligned}
\left\langle A^{2}\right\rangle & =\mathcal{A}^{2}\left[\left(1+\cos ^{2} \iota\right)^{2}\left\langle F_{+}^{2}\right\rangle+4 \cos ^{2} \iota\left\langle F_{\times}^{2}\right\rangle\right], \\
\left\langle F_{+}^{2}\right\rangle & =\frac{1}{4}\left(\cos ^{2} 2 \psi\left\langle D_{+}^{2}\right\rangle-\sin 4 \psi\left\langle D_{+} D_{\times}\right\rangle+\sin ^{2} 2 \psi\left\langle D_{\times}^{2}\right\rangle\right), \\
\left\langle F_{\times}^{2}\right\rangle & =\frac{1}{4}\left(\cos ^{2} 2 \psi\left\langle D_{\times}^{2}\right\rangle+\sin 4 \psi\left\langle D_{+} D_{\times}\right\rangle+\sin ^{2} 2 \psi\left\langle D_{+}^{2}\right\rangle\right) .
\end{aligned}
$$

where

$$
\begin{aligned}
\left\langle D_{+}^{2}\right\rangle & =b_{1}^{2}+b_{2}^{2}, \\
\left\langle D_{\times}^{2}\right\rangle & =b_{3}^{2}+b_{4}^{2}, \\
\left\langle D_{+} D_{\times}\right\rangle & =-2\left(b_{1} b_{3}+b_{2} b_{4}\right),
\end{aligned}
$$

and

$$
\begin{aligned}
b_{1}= & \frac{\sqrt{3}}{8}\left((3+\cos 2 \theta) \sin \theta_{s} \sin 2\left(\phi-\phi_{s}\right)+2 \sin \left(\phi-\phi_{s}\right) \sin 2 \theta \cos \theta_{s}\right), \\
b_{2}= & \frac{\sqrt{3}}{32}\left(3+\cos 2\left(\phi-\phi_{s}\right)\left(9+\cos 2 \theta\left(3-\cos 2 \theta_{s}\right)\right)+6 \cos 2 \theta_{s} \sin ^{2}\left(\phi-\phi_{s}\right)\right. \\
& \left.-6 \cos 2 \theta \cos ^{2} \theta_{s}+4 \cos \left(\phi-\phi_{s}\right) \sin 2 \theta \sin 2 \theta_{s}\right), \\
b_{3}= & \frac{\sqrt{3}}{2}\left(\cos 2\left(\phi-\phi_{s}\right) \cos \theta \sin \theta_{s}+\cos \left(\phi-\phi_{s}\right) \sin \theta \cos \theta_{s}\right), \\
b_{4}= & \frac{\sqrt{3}}{8}\left(\left(3-\cos 2 \theta_{s}\right) \cos \theta \sin 2\left(\phi_{s}-\phi\right)+2 \sin \left(\phi_{s}-\phi\right) \sin \theta \sin 2 \theta_{s}\right) .
\end{aligned}
$$

The average amplitude calculated by Eqs. (D3)-(D7) is consistent with Eqs. (19)-(21), with $0.1 \% \sim 1 \%$ of relative uncertainty.

\section{Appendix E: Coordinate transformation}

The transformation of the source position from the ecliptic coordinates $(\beta, \lambda)$ to the detector coordinates $\left(\theta_{S}, \phi_{S}\right)$ and $\left(\theta_{S}^{\prime}, \phi_{S}^{\prime}\right)$ of the TianQin (TQ) and TQ II is described by the following formula:

$$
\begin{gathered}
\left(\begin{array}{c}
d \sin \theta_{S} \cos \phi_{S} \\
d \sin \theta_{S} \sin \phi_{S} \\
d \cos \theta_{S}
\end{array}\right)=R_{x}\left(\theta=120^{\circ}-90^{\circ}\right) R_{z}\left(\theta=-4.7^{\circ}-90^{\circ}\right)\left(\begin{array}{c}
d \cos \beta \cos \lambda \\
d \cos \beta \sin \lambda \\
d \sin \beta
\end{array}\right) \\
\text { and }\left(\begin{array}{c}
d \sin \theta_{S}^{\prime} \cos \phi_{S}^{\prime} \\
d \sin \theta_{S}^{\prime} \sin \phi_{S}^{\prime} \\
d \cos \theta_{S}^{\prime}
\end{array}\right)=R_{y}\left(\theta=90^{\circ}\right) R_{x}\left(\theta=120^{\circ}-90^{\circ}\right) R_{z}\left(\theta=-4.7^{\circ}-90^{\circ}\right)\left(\begin{array}{c}
d \cos \beta \cos \lambda \\
d \cos \beta \sin \lambda \\
d \sin \beta
\end{array}\right),
\end{gathered}
$$

where the rotation matrices are

$$
R_{x}(\theta)=\left(\begin{array}{ccc}
1 & 0 & 0 \\
0 & \cos \theta & \sin \theta \\
0 & -\sin \theta & \cos \theta
\end{array}\right), R_{y}(\theta)=\left(\begin{array}{ccc}
\cos \theta & 0 & -\sin \theta \\
0 & 1 & 0 \\
\sin \theta & 0 & \cos \theta
\end{array}\right), \text { and } R_{z}(\theta)=\left(\begin{array}{ccc}
\cos \theta & \sin \theta & 0 \\
-\sin \theta & \cos \theta & 0 \\
0 & 0 & 1
\end{array}\right) .
$$

[1] B. P. Abbott et al. (LIGO Scientific Collaboration and Virgo Collaboration), Phys. Rev. Lett. 116, 061102 
[2] A. Einstein, Sitzungsberichte der Königlich Preußischen Akademie der Wissenschaften (Berlin , 688 (1916).

[3] B. P. Abbott et al. (LIGO Scientific Collaboration and Virgo Collaboration), Phys. Rev. X 6, 041015 (2016).

[4] B. P. Abbott et al. (LIGO Scientific Collaboration and Virgo Collaboration), Phys. Rev. X 9, 031040 (2019).

[5] The LIGO Scientific Collaboration, the Virgo Collaboration, et al., arXiv e-prints , arXiv:2004.08342 (2020), arXiv:2004.08342 [astro-ph.HE].

[6] B. P. Abbott et al., Astrophysical Journal 892, L3 (2020), arXiv:2001.01761 [astro-ph.HE].

[7] R. Abbott et al., Astrophysical Journal 896, L44 (2020), arXiv:2006.12611 [astro-ph.HE].

[8] S. T. McWilliams, R. Caldwell, K. Holley-Bockelmann, S. L. Larson, and M. Vallisneri, arXiv e-prints (2019), arXiv:1903.04592 [astro-ph.HE].

[9] M. Kamionkowski and E. D. Kovetz, ARA\&A 54, 227 (2016), arXiv:1510.06042.

[10] Z. Arzoumanian et al., Astrophysical Journal 859, 47 (2018), arXiv:1801.02617 [astro-ph.HE].

[11] R. M. Shannon et al., Science 349, 1522 (2015), arXiv:1509.07320.

[12] P. Amaro-Seoane et al., arXiv e-prints (2017), arXiv:1702.00786 [astro-ph.IM].

[13] J. Luo et al. (TianQin), Class. Quant. Grav. 33, 035010 (2016), arXiv:1512.02076 [astro-ph.IM].

[14] A. Klein, E. Barausse, A. Sesana, A. Petiteau, E. Berti, S. Babak, J. Gair, S. Aoudia, I. Hinder, F. Ohme, and B. Wardell, Phys. Rev. D 93, 024003 (2016).

[15] H.-T. Wang, Z. Jiang, A. Sesana, E. Barausse, S.-J. Huang, Y.-F. Wang, W.-F. Feng, Y. Wang, Y.-M. Hu, J. Mei, and J. Luo, Phys. Rev. D 100, 043003 (2019), arXiv:1902.04423 [astro-ph.HE].

[16] J. Magorrian, S. Tremaine, D. Richstone, R. Bender, G. Bower, A. Dressler, S. M. Faber, K. Gebhardt, R. Green, C. Grillmair, J. Kormendy, and T. Lauer, Astronomical Journal 115, 2285 (1998), astro-ph/9708072.

[17] D. Lynden-Bell, Nature 223, 690 (1969).

[18] W.-F. Feng, H.-T. Wang, X.-C. Hu, Y.-M. Hu, and Y. Wang, Phys. Rev. D 99, 123002 (2019), arXiv:1901.02159 [astro-ph.IM].

[19] S. Babak, J. Gair, A. Sesana, E. Barausse, C. F. Sopuerta, C. P. L. Berry, E. Berti, P. Amaro-Seoane, A. Petiteau, and A. Klein, Phys. Rev. D 95, 103012 (2017).

[20] H.-M. Fan, Y.-M. Hu, E. Barausse, A. Sesana, J.D. Zhang, X. Zhang, T.-G. Zi, and J. Mei, arXiv e-prints , arXiv:2005.08212 (2020), arXiv:2005.08212 [astro-ph.HE].

[21] A. Lamberts, S. Garrison-Kimmel, P. F. Hopkins, E. Quataert, J. S. Bullock, C. A. Faucher-Giguère, A. Wetzel, D. Kereš, K. Drango, and R. E. Sand erson, MNRAS 480, 2704 (2018), arXiv:1801.03099 [astroph.GA].

[22] M. Y. M. Lau, I. Mandel, A. Vigna-Gómez, C. J. Neijssel, S. Stevenson, and A. Sesana, MNRAS 492, 3061 (2020), arXiv:1910.12422 [astro-ph.HE].

[23] V. Korol, E. M. Rossi, P. J. Groot, G. Nelemans, S. Toonen, and A. G. A. Brown, MNRAS 470, 1894 (2017), arXiv:1703.02555 [astro-ph.HE].

[24] T. Robson, N. J. Cornish, N. Tamanini, and S. Toonen, Phys. Rev. D 98, 064012 (2018), arXiv:1806.00500 [grqc].

[25] J. D. Romano and N. J. Cornish, Living Reviews in Relativity 20, 2 (2017), arXiv:1608.06889 [gr-qc].
[26] Z. C. Liang et al., "Science with the tianqin observatory: Preliminary results on stochastic gravitational wave background," (2020), in prep.

[27] V. Korol, O. Koop, and E. M. Rossi, Astrophysical Journal 866, L20 (2018), arXiv:1808.05959 [astroph.HE].

[28] E. Berti, A. Buonanno, and C. M. Will, Phys. Rev. D 71, 084025 (2005), gr-qc/0411129.

[29] C. Shi, J. Bao, H. Wang, J.-d. Zhang, Y. Hu, A. Sesana, E. Barausse, J. Mei, and J. Luo, Phys. Rev. D 100, 044036 (2019), arXiv:1902.08922 [gr-qc].

[30] J. Bao, C. Shi, H. Wang, J.-d. Zhang, Y. Hu, J. Mei, and J. Luo, Phys. Rev. D 100, 084024 (2019), arXiv:1905.11674 [gr-qc].

[31] N. Tamanini and C. Danielski, arXiv e-prints (2018), arXiv:1812.04330 [astro-ph.EP].

[32] G. Nelemans, L. R. Yungelson, and S. F. Portegies Zwart, A\&A 375, 890 (2001), astro-ph/0105221.

[33] S. Yu and C. S. Jeffery, A\&A 521, A85 (2010), arXiv:1007.4267 [astro-ph.SR].

[34] K. Breivik, S. C. Coughlin, M. Zevin, C. L. Rodriguez, K. Kremer, C. S. Ye, J. J. Andrews, M. Kurkowski, M. C. Digman, S. L. Larson, and F. A. Rasio, arXiv e-prints , arXiv:1911.00903 (2019), arXiv:1911.00903 [astro-ph.HE].

[35] K. A. Postnov and L. R. Yungelson, Living Reviews in Relativity 17, 3 (2014).

[36] K. Belczynski, V. Kalogera, and T. Bulik, The Astrophysical Journal 572, 407 (2002).

[37] G. Nelemans, L. R. Yungelson, S. F. Portegies Zwart, and F. Verbunt, A\&A 365, 491 (2001), astro$\mathrm{ph} / 0010457$.

[38] T. R. Marsh, G. Nelemans, and D. Steeghs, MNRAS 350, 113 (2004), astro-ph/0312577.

[39] J.-E. Solheim, PASP 122, 1133 (2010).

[40] T. M. Tauris, Phys. Rev. Lett 121, 131105 (2018), arXiv:1809.03504 [astro-ph.SR].

[41] L. Bildsten, K. J. Shen, N. N. Weinberg, and G. Nelemans, APJL 662, L95 (2007), astro-ph/0703578.

[42] R. F. Webbink, APJ 277, 355 (1984).

[43] I. Iben, Jr. and A. V. Tutukov, APJS 54, 335 (1984).

[44] A. L. Piro, APJL 740, L53 (2011), arXiv:1108.3110 [astro-ph.SR].

[45] J. Fuller and D. Lai, APJL 756, L17 (2012), arXiv:1206.0470 [astro-ph.SR].

[46] S. Dall'Osso and E. M. Rossi, MNRAS 443, 1057 (2014), arXiv:1308.1664 [astro-ph.HE].

[47] B. Mckernan and K. E. S. Ford, Monthly Notices of the Royal Astronomical Society 463, 2039 (2016).

[48] T. B. Littenberg and N. Yunes, Classical and Quantum Gravity 36, 095017 (2019), arXiv:1811.01093 [gr-qc].

[49] A. Cooray and N. Seto, Phys. Rev. D 69, 103502 (2004), astro-ph/0311054.

[50] M. Benacquista and K. Holley-Bockelmann, APJ 645, 589 (2006), astro-ph/0504135.

[51] M. R. Adams, N. J. Cornish, and T. B. Littenberg, Phys. Rev. D 86, 124032 (2012), arXiv:1209.6286 [grqc].

[52] V. Korol, E. M. Rossi, and E. Barausse, MNRAS 483, 5518 (2019), arXiv:1806.03306.

[53] M. J. C. Wilhelm, V. Korol, E. M. Rossi, and E. D'Onghia, arXiv e-prints , arXiv:2003.11074 (2020), arXiv:2003.11074 [astro-ph.GA].

[54] J. H. Steffen, D.-H. Wu, and S. L. Larson, arXiv e- 
prints (2018), arXiv:1812.03438 [astro-ph.EP].

[55] C. Danielski, V. Korol, N. Tamanini, and E. M. Rossi, A\&A 632, A113 (2019), arXiv:1910.05414 [astroph.EP].

[56] J. L. Yiming Hu, Jianwei Mei, Chinese Science Bulletin (2019).

[57] B.-B. Ye, X. Zhang, M.-Y. Zhou, Y. Wang, H.-M. Yuan, D. Gu, Y. Ding, J. Zhang, J. Mei, and J. Luo, International Journal of Modern Physics D (2019).

[58] Y. M. Hu, J. Mei, and J. Luo, National Science Review 4, $683(2017)$

[59] X.-C. Hu, X.-H. Li, Y. Wang, W.-F. Feng, M.-Y. Zhou, Y.-M. Hu, S.-C. Hu, J.-W. Mei, and C.-G. Shao, Classical and Quantum Gravity 35, 095008 (2018), arXiv:1803.03368 [gr-qc].

[60] S. Liu, Y.-M. Hu, J.-d. Zhang, and J. Mei, Phys. Rev. D 101, 103027 (2020), arXiv:2004.14242 [astro-ph.HE].

[61] N. Xie et al., "Detecting extra polarization of GW with TianQin," (2020), in prep.

[62] D. Liang, Y. Gong, A. J. Weinstein, C. Zhang, and C. Zhang, Phys. Rev. D 99, 104027 (2019), arXiv:1901.09624 [gr-qc].

[63] C. Zhang, Q. Gao, Y. Gong, B. Wang, A. J. Weinstein, and C. Zhang, arXiv e-prints, arXiv:2003.01441 (2020), arXiv:2003.01441 [gr-qc].

[64] V. Korol, S. Toonen, A. Klein, V. Belokurov, F. Vincenzo, R. Buscicchio, D. Gerosa, C. J. Moore, E. Roebber, E. M. Rossi, and A. Vecchio, arXiv e-prints , arXiv:2002.10462 (2020), arXiv:2002.10462 [astroph.GA].

[65] E. Roebber, R. Buscicchio, A. Vecchio, C. J. Moore, A. Klein, V. Korol, S. Toonen, D. Gerosa, J. Goldstein, S. M. Gaebel, and T. E. Woods, arXiv e-prints , arXiv:2002.10465 (2020), arXiv:2002.10465 [astroph.GA].

[66] G. Nelemans, L. R. Yungelson, and S. F. Portegies Zwart, MNRAS 349, 181 (2004), astro-ph/0312193.

[67] S. Nissanke, M. Vallisneri, G. Nelemans, and T. A. Prince, Astrophysical Journal 758, 131 (2012), arXiv:1201.4613.

[68] W. R. Brown, M. Kilic, A. Kosakowski, and A. Gianninas, The Astrophysical Journal 847, 10 (2017).

[69] D. Maoz, N. Hallakoun, and C. Badenes, MNRAS 476, 2584 (2018), arXiv:1801.04275 [astro-ph.SR].

[70] G. Ramsay, M. J. Green, T. R. Marsh, T. Kupfer, E. Breedt, V. Korol, P. J. Groot, C. Knigge, G. Nelemans, D. Steeghs, P. Woudt, and A. Aungwerojwit, A\&A 620, A141 (2018), arXiv:1810.06548 [astroph.SR].

[71] K. B. Burdge, M. W. Coughlin, J. Fuller, T. Kupfer, E. C. Bellm, L. Bildsten, M. J. Graham, D. L. Kaplan, J. v. Roestel, R. G. Dekany, D. A. Duev, M. Feeney, M. Giomi, G. Helou, S. Kaye, R. R. Laher, A. A. Mahabal, F. J. Masci, R. Riddle, D. L. Shupe, M. T. Soumagnac, R. M. Smith, P. Szkody, R. Walters, S. R. Kulkarni, and T. A. Prince, Nature 571, 528 (2019), arXiv:1907.11291 [astro-ph.SR].

[72] K. B. Burdge, J. Fuller, E. S. Phinney, J. van Roestel, A. Claret, E. Cukanovaite, N. P. Gentile Fusillo, M. W. Coughlin, D. L. Kaplan, T. Kupfer, P.-E. Tremblay, R. G. Dekany, D. A. Duev, M. Feeney, R. Riddle, S. R. Kulkarni, and T. A. Prince, Astrophysical Journal 886, L12 (2019), arXiv:1910.11389 [astro-ph.SR].

[73] M. W. Coughlin, K. Burdge, E. Sterl Phinney, J. van
Roestel, E. C. Bellm, R. G. Dekany, A. Delacroix, D. A. Duev, M. Feeney, M. J. Graham, S. R. Kulkarni, T. Kupfer, R. R. Laher, F. J. Masci, T. A. Prince, R. Riddle, P. Rosnet, R. Smith, E. Serabyn, and R. Walters, MNRAS 494, L91 (2020), arXiv:2004.00456 [astro-ph.HE].

[74] W. R. Brown, M. Kilic, A. Bedard, A. Kosakowski, and P. Bergeron, arXiv e-prints , arXiv:2004.00641 (2020), arXiv:2004.00641 [astro-ph.SR].

[75] A. Stroeer and A. Vecchio, Classical and Quantum Gravity 23, S809 (2006), arXiv:astro-ph/0605227 [astro-ph].

[76] T. Kupfer, S. Shah, G. Nelemans, T. R. Marsh, G. Ramsay, P. J. Groot, D. T. H. Steeghs, and E. M. Rossi, MNRAS 480, 302 (2018).

[77] Gaia Collaboration, A. G. A. Brown, A. Vallenari, T. Prusti, J. H. J. de Bruijne, C. Babusiaux, and C. A. L. Bailer-Jones, ArXiv e-prints (2018), arXiv:1804.09365.

[78] T. E. Strohmayer, Astrophysical Journal 627, 920 (2005), arXiv:astro-ph/0504150 [astro-ph].

[79] G. H. A. Roelofs, A. Rau, T. R. Marsh, D. Steeghs, P. J. Groot, and G. Nelemans, Astrophysical Journal 711, L138 (2010), arXiv:1003.0658 [astro-ph.SR].

[80] S. Toonen, G. Nelemans, and S. Portegies Zwart, A\&A 546, A70 (2012), arXiv:1208.6446 [astro-ph.HE].

[81] S. Toonen, M. Hollands, B. T. Gänsicke, and T. Boekholt, A\&A 602, A16 (2017), arXiv:1703.06893 [astro-ph.SR].

[82] S. F. Portegies Zwart and F. Verbunt, A\&A 309, 179 (1996).

[83] P. Kroupa, C. A. Tout, and G. Gilmore, MNRAS 262, 545 (1993).

[84] G. Duchêne and A. Kraus, ARA\&A 51, 269 (2013), arXiv:1303.3028 [astro-ph.SR].

[85] H. A. Abt, ARA\&A 21, 343 (1983).

[86] D. C. Heggie, MNRAS 173, 729 (1975).

[87] G. Nelemans, F. Verbunt, L. R. Yungelson, and S. F. Portegies Zwart, A\&A 360, 1011 (2000), astro$\mathrm{ph} / 0006216$.

[88] S. Boissier and N. Prantzos, MNRAS 307, 857 (1999), astro-ph/9902148.

[89] L. D. Landau and E. M. Lifshitz, The classical theory of fields; 2nd ed., Course of theoretical physics (Pergamon, London, 1962) trans. from the Russian.

[90] P. C. Peters and J. Mathews, Phys. Rev. 131, 435 (1963).

[91] C. Cutler, Phys. Rev. D 57, 7089 (1998).

[92] N. J. Cornish and L. J. Rubbo, Phys. Rev. D 67, 029905 (2003), arXiv:gr-qc/0209011 [gr-qc].

[93] T. Robson, N. J. Cornish, and C. Liu, Classical and Quantum Gravity 36, 105011 (2019), arXiv:1803.01944 [astro-ph.HE].

[94] L. S. Finn, Phys. Rev. D 46, 5236 (1992).

[95] C. Cutler and E. E. Flanagan, Phys. Rev. D 49, 2658 (1994).

[96] N. Cornish and T. Robson, Proceedings, 11th International LISA Symposium: Zurich, Switzerland, September 5-9, 2016, J. Phys. Conf. Ser. 840, 012024 (2017), arXiv:1703.09858 [astro-ph.IM].

[97] T. B. Littenberg and N. J. Cornish, Phys. Rev. D91, 084034 (2015), arXiv:1410.3852 [gr-qc].

[98] S. Shah, M. van der Sluys, and G. Nelemans, A\&A 544, A153 (2012), arXiv:1207.6770 [astro-ph.IM]. 
[99] T. B. Littenberg, S. L. Larson, G. Nelemans, and N. J. Cornish, MNRAS 429, 2361 (2013).

[100] M. Hannam, P. Schmidt, A. Bohé, L. Haegel, S. Husa, F. Ohme, G. Pratten, and M. Pürrer, Phys. Rev. Lett 113, 151101 (2014), arXiv:1308.3271 [gr-qc].

[101] N. Hallakoun and D. Maoz, arXiv e-prints (2019), arXiv:1905.00032 [astro-ph.SR].

[102] B. Wang and D. Liu, arXiv e-prints , arXiv:2005.01880 (2020), arXiv:2005.01880 [astro-ph.SR].

[103] I. D. Karachentsev and O. G. Kashibadze, Astrophysics 49, 3 (2006).

[104] W. R. Brown, M. Kilic, S. J. Kenyon, and A. Gianninas, Astrophysical Journal 824, 46 (2016), arXiv:1604.04269 [astro-ph.SR].

[105] E. Bellm, in The Third Hot-wiring the Transient Universe Workshop, edited by P. R. Wozniak, M. J. Gra- ham, A. A. Mahabal, and R. Seaman (2014) pp. 27-33, arXiv:1410.8185 [astro-ph.IM].

[106] LSST Science Collaboration, P. A. Abell, J. Allison, S. F. Anderson, J. R. Andrew, J. R. P. Angel, L. Armus, D. Arnett, S. J. Asztalos, T. S. Axelrod, and et al., arXiv e-prints (2009), arXiv:0912.0201 [astro-ph.IM].

[107] D. Steeghs, Nature Astronomy 1, 741 (2017).

[108] S. Bloemen, P. Groot, G. Nelemans, and M. KleinWolt, "The BlackGEM Array: Searching for Gravitational Wave Source Counterparts to Study UltraCompact Binaries," in Living Together: Planets, Host Stars and Binaries, Astronomical Society of the Pacific Conference Series, Vol. 496, edited by S. M. Rucinski, G. Torres, and M. Zejda (2015) p. 254.

[109] G. Nelemans, LISA Verification Binaries (2010). 